TRANSACTIONS OF THE

AMERICAN MATHEMATICAL SOCIETY

Volume 355, Number 12, Pages 4901-4932

S 0002-9947(03)03199-4

Article electronically published on July 28, 2003

\title{
FAMILIES OF NODAL CURVES ON PROJECTIVE THREEFOLDS AND THEIR REGULARITY VIA POSTULATION OF NODES
}

\author{
FLAMINIO FLAMINI
}

\begin{abstract}
The main purpose of this paper is to introduce a new approach to study families of nodal curves on projective threefolds. Precisely, given a smooth projective threefold $X$, a rank-two vector bundle $\mathcal{E}$ on $X$, and integers $k \geq 0, \delta>0$, denote by $\mathcal{V}_{\delta}(\mathcal{E}(k))$ the subscheme of $\mathbb{P}\left(H^{0}(\mathcal{E}(k))\right)$ parametrizing global sections of $\mathcal{E}(k)$ whose zero-loci are irreducible $\delta$-nodal curves on $X$. We present a new cohomological description of the tangent space $T_{[s]}\left(\mathcal{V}_{\delta}(\mathcal{E}(k))\right)$ at a point $[s] \in \mathcal{V}_{\delta}(\mathcal{E}(k))$. This description enables us to determine effective and uniform upper bounds for $\delta$, which are linear polynomials in $k$, such that the family $\mathcal{V}_{\delta}(\mathcal{E}(k))$ is smooth and of the expected dimension (regular, for short). The almost sharpness of our bounds is shown by some interesting examples. Furthermore, when $X$ is assumed to be a Fano or a Calabi-Yau threefold, we study in detail the regularity property of a point $[s] \in \mathcal{V}_{\delta}(\mathcal{E}(k))$ related to the postulation of the nodes of its zero-locus $C=V(s) \subset X$. Roughly speaking, when the nodes of $C$ are assumed to be in general position either on $X$, or on an irreducible divisor of $X$ having at worst log-terminal singularities or to lie on a l.c.i. and subcanonical curve in $X$, we find upper bounds on $\delta$ which are, respectively, cubic, quadratic and linear polynomials in $k$ ensuring the regularity of $\mathcal{V}_{\delta}(\mathcal{E}(k))$ at $[s]$. Finally, when $X=\mathbb{P}^{3}$, we also discuss some interesting geometric properties of the curves given by sections parametrized by $\mathcal{V}_{\delta}\left(\mathcal{E} \otimes \mathcal{O}_{X}(k)\right)$.
\end{abstract}

\section{INTRODUCTION}

The theory of families of singular curves with fixed invariants (e.g. geometric genus, singularity type, number of irreducible components, etc.) and which are contained in a projective variety $X$ has been extensively studied from the beginning of algebraic geometry, and it still receives a lot of attention, partially due to its connections with other fields of geometry and physics. Indeed, interest in this argument has grown essentially for two reasons: on the one hand, string theory in nuclear physics deals with enumerative geometry for rational curves contained in projective threefolds; on the other hand, the study of singular curves is naturally related to the hyperbolic geometry of complex projective varieties.

Nodal curves play a central role in the subject of singular curves. Families of irreducible and $\delta$-nodal curves on a given projective variety $X$ are usually called

Received by the editors June 25, 2002.

2000 Mathematics Subject Classification. Primary 14H10, 14J60; Secondary 14J30, 14J32, $14 \mathrm{~J} 45$.

Key words and phrases. Families of nodal curves, postulation of nodes, projective threefolds. The author is a member of Cofin GVA, EAGER and GNSAGA-INdAM. 
Severi varieties of irreducible, $\delta$-nodal curves in $X$. The terminology "Severi variety" is due to the classical case of families of nodal curves on $X=\mathbb{P}^{2}$, which was first studied by Severi (see [33]).

The case in which $X$ is a smooth projective surface has recently given rise to a huge amount of literature. (See, for example, [4], 6], 77, 99, 14], [17], [18, [29, [32], 36], just to mention a few. For a detailed chronological overview, the reader is referred for example to Section 2.3 in [13] and to its bibliography.) This interest has arisen not only because of the great interest in the subject, but also because for a Severi variety $V$ on an arbitrary projective variety $X$ there are several problems, such as non-emptiness, smoothness, irreducibility, and dimensional computation, as well as enumerative and moduli properties of the family of curves it parametrizes.

On the contrary, in higher dimensions only a few results are known. Precisely, on the one hand we have non-emptiness and enumerative results for some classes of varieties which are relevant for applications; on the other hand, some other results of non-emptiness and smoothness are given only for families of nodal curves in projective spaces (see, e.g., [1] and [32]).

Therefore, we feel some lack of systematic studies for what should be the next relevant case, from the point of view of algebraic geometry: families of nodal curves on projective threefolds.

The purpose of this paper is twofold: first, we introduce a new method to determine when a given (non-empty) Severi variety on a smooth projective threefold $X$ is smooth and of the expected dimension - regular, for short - at a given point (for details, see Definition 2.11); then, we apply this method to find geometric and numerical sufficient conditions for the regularity of Severi varieties.

In general, a natural approach to the regularity problem is to use the deformation theory of nodal curves in a smooth ambient variety. In the surface case, if $V_{\left|\mathcal{O}_{S}(D)\right|, \delta}$ denotes the Severi variety of irreducible $\delta$-nodal curves in the linear system $\left|\mathcal{O}_{S}(D)\right|$ on a smooth projective surface $S$, it is well known that, when $V_{\left|\mathcal{O}_{S}(D)\right|, \delta} \neq \emptyset$, its expected codimension in $\left|\mathcal{O}_{S}(D)\right|$ is $\delta$; moreover, if $[C] \in V_{\left|\mathcal{O}_{S}(D)\right|, \delta}$ parametrizes a curve $C$ whose set of nodes is denoted by $\Sigma$, then the Zariski tangent space at $[C]$ is

$$
T_{[C]}\left(V_{\left|\mathcal{O}_{S}(D)\right|, \delta}\right) \cong H^{0}\left(S, \mathcal{I}_{\Sigma / S} \otimes \mathcal{O}_{S}(D)\right) /\langle C\rangle,
$$

where $\mathcal{I}_{\Sigma / S}$ denotes the ideal sheaf of $\Sigma$ in $S$ (see, for example, 9]). Thus, since the relative obstruction space is contained in $H^{1}\left(S, \mathcal{I}_{\Sigma / S} \otimes \mathcal{O}_{S}(D)\right)$, the regularity of $V_{\left|\mathcal{O}_{S}(D)\right|, \delta}$ at $[C]$ holds iff $\Sigma$ imposes independent conditions on $\left|\mathcal{O}_{S}(D)\right|$; in particular, a sufficient condition for the regularity at $[C]$ is $h^{1}\left(S, \mathcal{I}_{\Sigma / S} \otimes \mathcal{O}_{S}(D)\right)=0$.

In the threefold case, one obtains a partially similar organization for curves which are zero-loci of sections of a rank-two vector bundle $\mathcal{F}$ on $X$, so that $\mathbb{P}\left(H^{0}(X, \mathcal{F})\right)$ (which plays the same role as $\left|\mathcal{O}_{S}(D)\right|$ ) somehow gives a projective space dominating a subvariety in which the curves move. As in the surface case, if $\mathcal{V}_{\delta}(\mathcal{F})$ denotes the subscheme of $\mathbb{P}\left(H^{0}(X, \mathcal{F})\right)$ parametrizing global sections whose zero-loci are irreducible and $\delta$-nodal curves in $X$, with a little abuse of terminology we shall always use the term Severi variety to refer to $\mathcal{V}_{\delta}(\mathcal{F})$. In several cases (e.g. when $\mathcal{F}$ is a stable and aCM rank-two vector bundle on $X$ (for definitions, see [8])) this is not an abuse, since $\mathcal{V}_{\delta}(\mathcal{F})$ actually parametrizes irreducible nodal curves on $X$ (see Lemma 4.3 in [8]).

Notation 0.1. In the sequel, we write $[s] \in \mathcal{V}_{\delta}(\mathcal{F})$ to indicate that the global section $s \in H^{0}(X, \mathcal{F})$ determines the corresponding point $[s]$ of the scheme $\mathcal{V}_{\delta}(\mathcal{F})$. We also 
denote by $C_{s}$ (or simply $C$, when this does not create ambiguity) the zero-locus of the given section $s$, i.e. $C=V(s) \subset X$.

As in the surface case, when $\mathcal{V}_{\delta}(\mathcal{F})$ is not empty its expected codimension is $\delta$ (see Proposition 2.10); however, if $[s] \in \mathcal{V}_{\delta}(\mathcal{F})$ and $\Sigma$ denotes the set of nodes of the corresponding curve $C_{s} \subset X$, then we have

$$
T_{[s]}\left(\mathcal{V}_{\delta}(\mathcal{F})\right) \supset H^{0}\left(X, \mathcal{I}_{\Sigma / X} \otimes \mathcal{F}\right)\langle s\rangle,
$$

so the latter is the tangent space at $[s]$ to a subscheme of $\mathbb{P}\left(H^{0}(X, \mathcal{F})\right)$ of a higher expected codimension.

We thus present a systematic study of equisingular deformation theory for the elements parametrized by $\mathcal{V}_{\delta}(\mathcal{F})$ on $X$. Specifically, in the following result, which is the core of the paper, we introduce a new cohomological description of the tangent space $T_{[s]}\left(\mathcal{V}_{\delta}(\mathcal{F})\right)$.

Theorem (see Theorem 3.4). Let $X$ be a smooth projective threefold. Let $\mathcal{F}$ be a globally generated rank-two vector bundle on $X$ and let $\delta$ be a positive integer. Let

$$
\begin{aligned}
& \mathcal{V}_{\delta}(\mathcal{F}):=\left\{[s] \in \mathbb{P}\left(H^{0}(\mathcal{F})\right) \mid C_{s}:=V(s) \subset X\right. \text { is irreducible } \\
&\text { with only } \delta \text { nodes as singularities }\} .
\end{aligned}
$$

Consider $[s] \in \mathcal{V}_{\delta}(\mathcal{F})$ and denote by $\Sigma$ the set of nodes of the corresponding curve $C_{s} \subset X$. Let

$$
\mathcal{P}:=\mathbb{P}_{X}(\mathcal{F}) \stackrel{\pi}{\longrightarrow} X
$$

be the projective space bundle together with its natural projection $\pi$ on $X$, and denote by $\mathcal{O}_{\mathcal{P}}(1)$ its tautological line bundle. Let $T_{C_{s}}^{1}$ be the first cotangent sheaf of $C_{s}$ (see (3.1)) and let

$$
\Sigma^{1}:=\mathbb{P}_{X}\left(T_{C_{s}}^{1}\right) \subset \mathcal{P}
$$

be the zero-dimensional subscheme of $\mathcal{P}$ of length $\delta$, determined by the surjection $\mathcal{F} \rightarrow T^{1} C_{s} \rightarrow 0$. Denote by $\mathcal{I}_{\Sigma^{1} / \mathcal{P}}$ the ideal sheaf of $\Sigma^{1}$ in $\mathcal{P}$. Then

(i) $\Sigma^{1}$ is a set of $\delta$ rational double points for the divisor $D_{s} \in\left|\mathcal{O}_{\mathcal{P}}(1)\right|$, corresponding to the given section $s \in H^{0}(X, \mathcal{F})$, and

(ii) the subsheaf of $\mathcal{F}$ defined by

$$
\mathcal{F}^{\Sigma}:=\pi_{*}\left(\mathcal{I}_{\Sigma^{1} / \mathcal{P}} \otimes \mathcal{O}_{\mathcal{P}}(1)\right)
$$

is such that its global sections (modulo the one-dimensional subspace $\langle s\rangle$ ) parametrize first-order deformations of $s \in H^{0}(X, \mathcal{F})$ which are equisingular.

In particular, we have

$$
\frac{H^{0}\left(X, \mathcal{F}^{\Sigma}\right)}{\langle s\rangle} \cong T_{[s]}\left(\mathcal{V}_{\delta}(\mathcal{F})\right) \subset T_{[s]}\left(\mathbb{P}\left(H^{0}(\mathcal{F})\right)\right) \cong \frac{H^{0}(X, \mathcal{F})}{\langle s\rangle}
$$

We want to briefly remark that the above result can be the starting point for the characterization of tangent spaces to such families on a smooth projective $n$-fold $Y$ with $n \geq 4$. The main difference from the threefold case is that one should work inside a suitable incidence variety $\mathcal{I} \subset \mathbb{P}_{Y}(\mathcal{F}) \times \mathbb{P}_{Y}\left(\mathcal{F}^{\vee}\right)$, where $\operatorname{rank}(\mathcal{F})=n-1$.

By using the above characterization of $T_{[s]}\left(\mathcal{V}_{\delta}(\mathcal{F})\right)$, we are able to determine geometric and numerical sufficient conditions for the regularity of $\mathcal{V}_{\delta}(\mathcal{F})$ at the point $[s]$. 
Indeed, we first prove the following:

Theorem (see Theorem 4.5). Let $X \subset \mathbb{P}^{r}$ be a smooth threefold with hyperplane bundle $\mathcal{O}_{X}(1)$. Let $\mathcal{E}$ be a globally generated rank-two vector bundle, and let $k \geq 0$ and $\delta>0$ be integers. If

$$
\delta \leq k+1,
$$

then $\mathcal{V}_{\delta}(\mathcal{E}(k))$ is smooth and of the expected dimension (i.e. regular) at each point.

Therefore, the above result determines sufficient conditions for $\mathcal{V}_{\delta}(\mathcal{E}(k))$ to be regular everywhere. Observe also that the bound $(*)$ is uniform, i.e. it does not depend on the postulation of the nodes of the curves related to the elements parametrized by $\mathcal{V}_{\delta}(\mathcal{E}(k))$; furthermore, the bound is almost sharp, as one can deduce from Example 3.2 in [1] and from our Remarks 5.21 and 5.38. We also stress that the above result generalizes the results of [1, mainly because our approach holds more generally for families of nodal curves on smooth projective threefolds, but also because, even in the case of $X=\mathbb{P}^{3}$, the main subject of [1], our bounds are effective and not asymptotic as Proposition 3.1 in [1].

After this, in $\S 5$ we focus on the case when $X$ is either a Fano or a Calabi-Yau threefold, and we "stratify" the regularity property in terms of the postulation of nodes. Precisely, by using the notion of local positivity of line bundles on $X$, the machinery of Seshadri constants as in [11, 12] and [24] and the fundamental tool of the Kawamata-Viehweg vanishing theorem, we determine some upper bounds for the number $\delta$, which are cubic polynomials in the integer $k$, such that if the $\delta$ nodes of a curve $C=V(s)$ are in very general position on $X$ (see Definition 5.8), then the point $[s]$ is regular for $\mathcal{V}_{\delta}(\mathcal{E}(k)$ ) (see Theorem [5.9] and Corollary 5.15). Furthermore, when the nodes of $C$ are assumed to be points in very general position on an irreducible divisor of $X$ having at worst log-terminal singularities or to lie on an l.c.i. and subcanonical curve in $X$, we determine upper bounds on $\delta$ which are, respectively, quadratic and linear polynomials in the integer $k$, implying the regularity of the point $[s]$ (see Theorems [5.25, 5.28, 5.36 and Corollaries [5.26 5.37).

We conclude the paper by focusing on the case $X=\mathbb{P}^{3}$ and studying interesting geometric properties of space curves determined by elements in $\mathcal{V}_{\delta}(\mathcal{E}(k))$.

The paper consists of six sections. In Section 1 we recall some terminology and notation. Section 2 contains fundamental definitions and technical details which are useful for our proofs. Section 3 contains our main result (see Theorem 3.4), which gives a cohomological description of the tangent space $T_{[s]}\left(\mathcal{V}_{\delta}(\mathcal{F})\right)$. In Section 4 , we prove our uniform and effective result for the regularity of $\mathcal{V}_{\delta}(\mathcal{F})$ at each point (see Theorem 4.5. Section 5 is devoted to the study of the regularity property of $\mathcal{V}_{\delta}(\mathcal{F})$ in terms of the postulation of nodes of the zero-loci of the elements it parametrizes. We conclude with Section 6, where we consider some geometric properties and biliaison relations of space curves determined by elements in $\mathcal{V}_{\delta}(\mathcal{F})$.

\section{Notation AND PRELIMINARIES}

We work in the category of algebraic $\mathbb{C}$-schemes. $Y$ is a $m$-fold if it is a reduced, irreducible and non-singular scheme of finite type and of dimension $m$. If $m=1$, then $Y$ is a (smooth) curve; $m=2$ and 3 are the cases of a (non-singular) surface and threefold, respectively. If $Z$ is a closed subscheme of a scheme $Y, \mathcal{I}_{Z / Y}$ denotes 
the ideal sheaf of $Z$ in $Y, \mathcal{N}_{Z / Y}$ the normal sheaf of $Z$ in $Y$, and $\mathcal{N}_{Z / Y}^{\vee} \cong \mathcal{I}_{Z / Y} / \mathcal{I}_{Z / Y}^{2}$ the conormal sheaf of $Z$ in $Y$. As usual, $h^{i}(Y,-):=\operatorname{dim} H^{i}(Y,-)$.

Given a projective scheme $Y, \omega_{Y}$ denotes its dualizing sheaf. When $Y$ is a smooth variety, then $\omega_{Y}$ coincides with its canonical bundle and $K_{Y}$ denotes a canonical divisor such that $\omega_{Y} \cong \mathcal{O}_{Y}\left(K_{Y}\right)$. Furthermore, $\mathcal{T}_{Y}$ denotes its tangent bundle and $\Omega_{Y}^{1}$ denotes its cotangent bundle.

If $D$ is a reduced curve, $p_{a}(D)=h^{1}\left(\mathcal{O}_{D}\right)$ denotes its arithmetic genus and $g(D)=$ $p_{g}(D)$ denotes its geometric genus, the arithmetic genus of its normalization.

Consider a projective $m$-fold $Y$. Div $(Y)$ denotes the set of (Cartier) divisors and $\sim$ the linear equivalence on $\operatorname{Div}(Y)$, whereas $\operatorname{Pic}(Y)$ denotes the Picard scheme of line bundles on $Y$. On the other hand, as in [19], $F_{1}(Y)$ denotes the free abelian group generated by the set of all integral curves in $Y$. Denote by $\cdot$ the intersection pairing on $Y$ and by $\equiv$ the numerical equivalence on $Y$. We have

$$
A^{1}(Y)=(\operatorname{Div}(Y) / \equiv) \otimes_{\mathbb{Z}} \mathbb{R} \quad \text { and } \quad A_{1}(Y)=\left(F_{1}(Y) / \equiv\right) \otimes_{\mathbb{Z}} \mathbb{R} .
$$

Recall that an element $B \in \operatorname{Div}(Y)$ is said to be nef, if $B \cdot D \geq 0$ for each irreducible curve $D$ on $Y$. A nef divisor $B$ is said to be big if $B^{m}>0$. By Kleiman's criterion (see, for example, [19]), a nef divisor $B$ is in the closure of the ample divisor cone $P^{0}(Y)$, which is the cone in $A^{1}(Y)$ generated by the ample divisors on $Y$. When $Y$ is a surface, the ample divisor cone is sometimes also denoted by $N^{+}(Y)$ (see, for example, [2] and [15]).

Let $Y$ be a projective $m$-fold and $\mathcal{E}$ a rank- $r$ vector bundle on $Y ; c_{i}(\mathcal{E})$ denotes the $i^{\text {th }}$ Chern class of $\mathcal{E}, 1 \leq i \leq r$. As in [20], Sect. II.7, $\mathbb{P}_{Y}(\mathcal{E})$ denotes the projective space bundle on $Y$, defined as $\operatorname{Proj}(\operatorname{Sym}(\mathcal{E}))$. There is a surjection $\pi^{*}(\mathcal{E}) \rightarrow \mathcal{O}_{\mathbb{P}_{Y}(\mathcal{E})}(1)$, where $\mathcal{O}_{\mathbb{P}_{Y}(\mathcal{E})}(1)$ is the tautological line bundle on $\mathbb{P}_{Y}(\mathcal{E})$ and where $\pi: \mathbb{P}_{Y}(\mathcal{E}) \rightarrow Y$ is the natural projection morphism. Recall that $\mathcal{E}$ is said to be an ample (resp. nef) vector bundle on $Y$ if $\mathcal{O}_{\mathbb{P}_{Y}(\mathcal{E})}(1)$ is an ample (resp. nef) line bundle on $\mathbb{P}_{Y}(\mathcal{E})$.

When $Y$ is a projective, normal variety of dimension $m$, the word divisor is used for Weil divisor, i.e. a formal linear combination of codimension-one subvarieties. A $\mathbb{Q}$-divisor $\left(\mathbb{R}\right.$-divisor, resp.) on $Y$ is a finite formal linear combination $D=\sum_{i} a_{i} D_{i}$ with rational (real, resp.) coefficients; when the coefficients are in $\mathbb{Z}, D$ is an integral divisor. $D$ is a $\mathbb{Q}$-Cartier divisor if some multiple of $D$ is an (integral) Cartier divisor (recall that, when $X$ is smooth, any $\mathbb{Q}$-divisor is $\mathbb{Q}$-Cartier). The round-up of $D$ and the integral part of $D$ are, respectively, the integral divisors $\lceil D\rceil=\sum_{i}\left\lceil a_{i}\right\rceil D_{i}$ and $[D]=\sum_{i}\left[a_{i}\right] D_{i}$, where, as usual, for $x \in \mathbb{Q}$ one denotes by $\lceil x\rceil$ the least integer greater than or equal to $x$ and by $[x]$ the greatest integer smaller than or equal to $x$. The fractional part of $D$ is $\{D\}=D-[D]$. Since there is a $\mathbb{Q}$-valued intersection theory for $\mathbb{Q}$-Cartier $\mathbb{Q}$-divisors, one can extend the notion of ampleness and nefness to $\mathbb{Q}$-divisors. Similarly, $D$ is big if $n D$ is integral and big, for some positive $n$. $D=\sum_{i} a_{i} D_{i}$ has simple normal crossings if each $D_{i}$ is smooth and if $D$ is defined in a neighborhood of any point by an equation in local analytic coordinates of the type $z_{1} \cdots z_{k}=0$, with $k \leq m$. A boundary divisor $\Delta$ is an effective divisor whose support has simple normal crossings and which is such that $[\Delta]=0$.

If $Y$ is a projective normal variety and $D$ is a $\mathbb{Q}$-divisor on $Y$, a log-resolution of the pair $(Y, D)$ is a proper birational mapping $\mu: Y^{\prime} \rightarrow Y$, where $Y^{\prime}$ is smooth and such that the divisor $\mu^{*}(D)+\operatorname{Exc}(\mu)$ has simple normal crossing support (here 
$\operatorname{Exc}(\mu)$ denotes the sum of the $\mu$-exceptional divisors). If $(Y, D)$ is such that $K_{Y}+D$ is $\mathbb{Q}$-Cartier and if $\mu$ is a log-resolution of the pair, then

$$
K_{Y^{\prime} / Y}-\mu^{*}(D):=K_{Y^{\prime}}-\mu^{*}\left(K_{Y}+D\right) \equiv \sum_{i} a_{i} E_{i},
$$

where the $E_{i}$ 's are distinct irreducible divisors (not necessarily all $\mu$-exceptional) and where the coefficients $a_{i}$ are called the discrepancies. The pair $(Y, D)$ is called log-terminal (resp. Kawamata log-terminal) if $a_{i}>-1$ for each $\mu$-exceptional $E_{i}$ (resp. for each $E_{i}$ ). Since $K_{Y^{\prime} / Y}$ is always $\mu$-exceptional, $Y$ is said to have at worst log-terminal singularities if the pair $(Y, 0)$ is log-terminal.

To conclude, we also recall one of the most important vanishing theorem for $\mathbb{Q}$-divisors, the Kawamata-Viehweg theorem, which will be frequently used in the sequel.

Theorem (Kawamata-Viehweg; see, for example, [28], page 146). Let $Y$ be a smooth projective variety of dimensione $m$, and let $D$ be a big and nef $\mathbb{Q}$-divisor whose fractional part has simple normal crossing support. Then

$$
H^{i}\left(Y, \mathcal{O}_{Y}\left(K_{Y}+\lceil D\rceil\right)=(0), \text { for } i>0 .\right.
$$

When $Y$ is a surface, the same conclusion holds even without the hypothesis on the fractional part of $D$.

\section{BASIC DEFINITIONS AND FUNDAMENTAL PROPERTIES}

In this section we introduce some fundamental definitions and remarks concerning the study of families of curves on smooth projective threefolds. For generalities, the reader is referred to [35], Chapter IV.

Definition 2.1. Let $X$ be a smooth projective threefold and let $\mathcal{F}$ be a rank-two vector bundle on $X$. Let $s$ be a global section of $\mathcal{F}$. The zero-locus of $s$, denoted by $V(s)$, is the closed subscheme of $X$ defined by the exact sequence

$$
\mathcal{F}^{\vee} \stackrel{s^{\vee}}{\longrightarrow} \mathcal{O}_{X} \rightarrow \mathcal{O}_{V(s)} \rightarrow 0,
$$

where $s^{\vee}$ is the dual map of the section $s$.

If $\operatorname{codim}_{X}(V(s))=2$, then $\operatorname{Ker}\left(s^{\vee}\right)=L^{\vee}$ is a line bundle on $X$ such that $c_{1}(\mathcal{F})=\bigwedge^{2}(\mathcal{F}) \cong L$. This yields the Koszul sequence of $(\mathcal{F}, s)$ :

$$
0 \rightarrow \mathcal{O}_{X} \rightarrow \mathcal{F} \rightarrow \mathcal{I}_{V(s)} \otimes L \rightarrow 0 .
$$

Remark 2.3. When Pic $(X) \cong \mathbb{Z}$ (e.g., $X=\mathbb{P}^{3}$ or $X$ is either a prime Fano or a complete intersection Calabi-Yau threefold) one can use this isomorphism to identify line bundles on $X$ with integers. In particular, if $A$ denotes the ample generator class of $\operatorname{Pic}(X)$ over $\mathbb{Z}$ and if $\mathcal{F}$ is a rank-two vector bundle on $X$ such that $c_{1}(\mathcal{F})=n A$, we can also write $c_{1}(\mathcal{F})=n$ with no ambiguity.

We recall well-known results concerning the correspondence between curves and global sections of vector bundles on a smooth projective threefold.

Theorem 2.4 (Serre). Let $X$ be a smooth projective threefold. A curve $D \subset X$ occurs as the zero-locus of a global section of a rank-two vector bundle $\mathcal{F}$ on $X$ if and only if $D$ is a locally complete intersection and its dualizing sheaf $\omega_{D}$ is 
isomorphic to the restriction to $D$ of $\omega_{X} \otimes M$, for some line bundle $M$ on $X$ such that

$$
h^{1}\left(X, M^{\vee}\right)=h^{2}\left(X, M^{\vee}\right)=0 .
$$

Furthermore, such a curve $D$ is a complete intersection in $X$ iff $\mathcal{F}$ splits.

Proposition 2.6. Let $X$ be a smooth projective threefold and let $\mathcal{F}$ be a rank-two vector bundle on $X$. If $\mathcal{F}$ is globally generated, then the zero-locus of a general section, if not empty, is non-singular of codimension two.

Proof. Particular case of standard results on generic morphisms of vector bundles; see e.g. [22].

From what we recalled above, if $X$ is a smooth projective threefold and $\mathcal{F}$ is a globally generated rank-two vector bundle on $X$, it is not restrictive to assume that the zero-locus of the general section of $\mathcal{F}$ is a smooth, irreducible curve $D$ in $X$. By the Koszul sequence (2.2), we find the geometric genus of $D$ in terms of the Chern classes of $\mathcal{F}$ and the invariants of $X$. Precisely,

$$
2 g(D)-2=2 p_{a}(D)-2=\operatorname{deg}\left(c_{1}(\mathcal{F}) \otimes \omega_{X} \otimes \mathcal{O}_{D}\right) .
$$

This integer is easily computable when, for example, $X$ is a general complete intersection threefold. In particular, when $X=\mathbb{P}^{3}$, by Remark 2.3 if we put $c_{i}=c_{i}(\mathcal{F}) \in \mathbb{Z}$, we have

$$
\operatorname{deg}(D)=c_{2} \text { and } g(D)=p_{g}(D)=\frac{1}{2}\left(c_{2}\left(c_{1}-4\right)\right)+1,
$$

i.e. $D$ is subcanonical of level $\left(c_{1}-4\right)$.

Now take $\mathbb{P}\left(H^{0}(\mathcal{F})\right)$; from our assumptions on $\mathcal{F}$, the general point of this projective space parametrizes a global section whose zero-locus is a smooth, irreducible curve in $X$. Given a positive integer $\delta$, one can consider the subset

$$
\begin{aligned}
\mathcal{V}_{\delta}(\mathcal{F}):= & \left\{[s] \in \mathbb{P}\left(H^{0}(\mathcal{F})\right) \mid C_{s}:=V(s) \subset X\right. \text { is irreducible } \\
& \text { with only } \delta \text { nodes as singularities }\}
\end{aligned}
$$

therefore, any element of $\mathcal{V}_{\delta}(\mathcal{F})$ determines a curve in $X$ whose arithmetic genus $p_{a}\left(C_{s}\right)$ is given by (2.7) and whose geometric genus is $g=p_{a}\left(C_{s}\right)-\delta$.

$\mathcal{V}_{\delta}(\mathcal{F})$ is a locally closed subscheme of the projective space $\mathbb{P}\left(H^{0}(\mathcal{F})\right)$, and it is usually called the Severi variety of global sections of $\mathcal{F}$ whose zero-loci are irreducible, $\delta$-nodal curves in $X$ (see [1]); this is because such schemes are the natural generalization of the (classical) Severi varieties of irreducible and $\delta$-nodal curves in linear systems on smooth, projective surfaces (see [6], 4], [9], [14, [17, [18, [29], [32] and [33], just to mention a few).

Proposition 2.10. Let $X$ be a smooth projective threefold, $\mathcal{F}$ a globally generated rank-two vector bundle on $X$, and $\delta$ a positive integer. Then

$$
\operatorname{expdim}\left(\mathcal{V}_{\delta}(\mathcal{F})\right)= \begin{cases}h^{0}(X, \mathcal{F})-1-\delta, & \text { if } \delta \leq h^{0}(X, \mathcal{F})-1=\operatorname{dim}\left(\mathbb{P}\left(H^{0}(\mathcal{F})\right)\right), \\ -1, & \text { if } \delta \geq h^{0}(X, \mathcal{F}) .\end{cases}
$$

Proof. If $\mathcal{V}_{\delta}(\mathcal{F})=\emptyset$, then $\operatorname{dim}\left(\mathcal{V}_{\delta}(\mathcal{F})\right)=-1$. On the other hand, when $\mathcal{V}_{\delta}(\mathcal{F}) \neq \emptyset$, consider the set

$$
\mathcal{U}_{\delta} \subset \mathbb{P}\left(H^{0}(\mathcal{F})\right) \times\left(X^{\delta} \backslash \bigcup_{1 \leq i \neq j \leq \delta} \Delta_{i, j}\right)
$$


where $X^{\delta}$ is the $\delta$-Cartesian product of $X, \Delta_{i, j}$ are the diagonals in $X^{\delta}$, and

$$
\begin{aligned}
\mathcal{U}_{\delta}:=\left\{\left([s] ; p_{1}, \ldots, p_{\delta}\right) \mid\right. & C=V(s) \subset X \text { is an irreducible curve } \\
& \text { with only nodes at the } \left.p_{i}\right\} .
\end{aligned}
$$

Since $X$ is smooth, for an arbitrary $p \in X$ we consider $U=U_{p}$, an affine open subscheme of $X$ containing $p$, with $\left(x_{1}^{(p)}, x_{2}^{(p)}, x_{3}^{(p)}\right)$ local coordinates in $U_{p}$, such that $\left.s\right|_{U_{p}}=\left(f_{1}^{(p)}, f_{2}^{(p)}\right)$, where $f_{i}^{(p)} \in \mathcal{O}_{X}\left(U_{p}\right)$. Define the closed subscheme

$$
\begin{aligned}
\mathcal{K}_{\delta}:= & \left\{\left([s] ; p_{1}, \ldots, p_{\delta}\right) \in \mathbb{P}\left(H^{0}(\mathcal{F})\right) \times\left(X^{\delta} \backslash \bigcup_{1 \leq i \neq j \leq \delta} \Delta_{i, j}\right) \mid s\left(p_{i}\right)=0\right. \text { and } \\
& \left.\operatorname{rank}\left(J(s)\left(p_{i}\right)\right) \leq 1,1 \leq i \leq \delta\right\},
\end{aligned}
$$

where, for an arbitrary $p \in X, J(s)(p)$ is the Jacobian matrix of $s$ at the point $p$. By definition,

$$
\begin{aligned}
\mathcal{K}_{\delta}:= & \left\{\left([s] ; p_{1}, \ldots, p_{\delta}\right) \mid s\left(p_{i}\right)=\left(\left(\frac{\partial}{\partial x_{1}^{\left(p_{i}\right)}} \wedge \frac{\partial}{\partial x_{2}^{\left(p i_{i}\right)}}\right)(s)\right)\left(p_{i}\right)\right. \\
& \left.=\left(\left(\frac{\partial}{\partial x_{1}^{\left(p_{i}\right)}} \wedge \frac{\partial}{\partial x_{3}^{\left(p_{i}\right)}}\right)(s)\right)\left(p_{i}\right)=\left(\left(\frac{\partial}{\partial x_{2}^{\left(p_{i}\right)}} \wedge \frac{\partial}{\partial x_{3}^{\left(p_{i}\right)}}\right)(s)\right)\left(p_{i}\right)=0,1 \leq i \leq \delta\right\} .
\end{aligned}
$$

Since $\mathcal{U}_{\delta}$ is contained in $\mathcal{K}_{\delta}$ as an open dense subscheme and since $\mathcal{K}_{\delta}$ is cut out by at most $4 \delta$ independent equations, then

$$
\begin{aligned}
\operatorname{dim}\left(\mathcal{U}_{\delta}\right)=\operatorname{dim}\left(\mathcal{K}_{\delta}\right) & \geq \operatorname{dim}\left(\mathbb{P}\left(H^{0}(\mathcal{F})\right) \times\left(X^{\delta} \backslash \bigcup_{1 \leq i \neq j \leq \delta} \Delta_{i, j}\right)\right)-4 \delta \\
& =h^{0}(X, \mathcal{F})-1+3 \delta-4 \delta=h^{0}(X, \mathcal{F})-1-\delta
\end{aligned}
$$

Denoting by $\pi_{1}$ the restriction to $\mathcal{U}_{\delta}$ of the projection onto the first factor of the product $\mathbb{P}\left(H^{0}(\mathcal{F})\right) \times X^{\delta}$, we have $\pi_{1}\left(\mathcal{U}_{\delta}\right)=\mathcal{V}_{\delta}(\mathcal{F})$. We conclude by observing that $\pi_{1}$ is finite onto its image.

Assumption. From now on, we shall use Notation 0.1. Moreover, given $X$ and $\mathcal{F}$ as in Proposition 2.10, we shall always assume $\mathcal{V}_{\delta}(\mathcal{F}) \neq \emptyset$ and $\delta \leq \min \left\{h^{0}(X, \mathcal{F})-\right.$ $\left.1, p_{a}(C)\right\}$ (the latter is because we want $C=V(s)$ to be irreducible).

By Proposition 2.10 we can state the following fundamental definition.

Definition 2.11. Let $[s] \in \mathcal{V}_{\delta}(\mathcal{F})$, with $\delta \leq \min \left\{h^{0}(X, \mathcal{F})-1, p_{a}(C)\right\}$. Then $[s]$ is said to be a regular point of $\mathcal{V}_{\delta}(\mathcal{F})$ if

(i) $[s] \in \mathcal{V}_{\delta}(\mathcal{F})$ is a smooth point, and

(ii) $\operatorname{dim}_{[s]}\left(\mathcal{V}_{\delta}(\mathcal{F})\right)=\operatorname{expdim}\left(\mathcal{V}_{\delta}(\mathcal{F})\right)=h^{0}(X, \mathcal{F})-1-\delta$, i.e.

$$
\operatorname{dim}_{[s]}\left(\mathcal{V}_{\delta}(\mathcal{F})\right)=\operatorname{dim}\left(\mathbb{P}\left(H^{0}(\mathcal{F})\right)\right)-\delta .
$$

The goal of the next section is to present a cohomological description of the tangent space $T_{[s]}\left(\mathcal{V}_{\delta}(\mathcal{F})\right)$ which will translate the regularity property of the point $[s] \in \mathcal{V}_{\delta}(\mathcal{F})$ into the surjectivity of some maps among vector spaces of sections of suitable sheaves on the threefold $X$.

\section{Description of the tangent Space $T_{[s]}\left(\mathcal{V}_{\delta}(\mathcal{F})\right)$ And Regularity}

As before, let $X$ be a smooth projective threefold and let $\mathcal{F}$ be a globally generated rank-two vector bundle on $X$. Let $\delta$ be a positive integer and consider $[s] \in \mathcal{V}_{\delta}(\mathcal{F})$. From now on in this section, let $C$ be the curve in $X$ which is the zero-locus of the given $s$, and let $\Sigma$ denote its set of $\delta$ nodes.

Since $C$ is a local complete intersection in $X$, its normal sheaf $\mathcal{N}_{C / X}$ is a ranktwo vector bundle (see [20]). Precisely, $\left.\mathcal{N}_{C / X} \cong \mathcal{F}\right|_{C}$. Let $T_{C}^{1}$ be the first cotangent 
sheaf of $C$, i.e. $T_{C}^{1} \cong \mathcal{E} x t^{1}\left(\Omega_{C}^{1}, \mathcal{O}_{C}\right)$, where $\Omega_{C}^{1}$ is the sheaf of Kähler differentials of the nodal curve $C$ (for details, see [27]). We have the exact sequence

$$
0 \rightarrow \mathcal{N}_{C}^{\prime} \rightarrow \mathcal{N}_{C / X} \stackrel{\gamma}{\rightarrow} T_{C}^{1} \rightarrow 0,
$$

where $\mathcal{N}_{C}^{\prime}$ is defined as the kernel of the natural surjection $\gamma$ (see, for example, 32]). Since nodal points are planar singularities, one has

i) $T_{C, p}^{1}=0$ and $\mathcal{N}_{C, p}^{\prime} \cong \mathcal{N}_{C / X, p} \cong \mathcal{O}_{C, p}^{\oplus 2}$, when $p \in C$ is a smooth point, and

ii) $T_{C, p}^{1} \cong \mathbb{C}$ and $\mathcal{N}_{C, p}^{\prime} \cong\left(\underline{m}_{p} \mathcal{O}_{C, p}\right) \oplus \mathcal{O}_{C, p}$, when $p$ is a node of $C\left(\underline{m}_{p}\right.$ denotes the maximal ideal at the point $p$ ).

Therefore, $T_{C}^{1}$ is a skyscraper sheaf supported on $\Sigma$, such that $T_{C}^{1} \cong \bigoplus_{i=1}^{\delta} \mathbb{C}_{(i)}$.

By using (3.1), the goal of this section is to construct a subsheaf $\mathcal{F}^{\Sigma} \subset \mathcal{F}$ fitting in the following exact diagram:

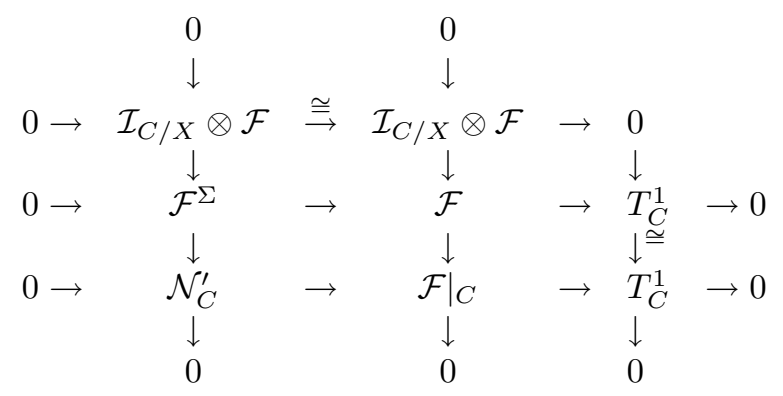

Observe that, from the commutativity of (3.2), $H^{0}\left(X, \mathcal{F}^{\Sigma}\right) /\langle s\rangle$ parametrizes the first-order deformations of the section $s$ in $H^{0}(X, \mathcal{F})$ which are equisingular; indeed, these are exactly the global sections of $\mathcal{F}$ which go to zero at $\Sigma$ in the composition

$$
\left.\mathcal{F} \rightarrow \mathcal{F}\right|_{C} \rightarrow T_{C}^{1} \cong \mathcal{O}_{\Sigma} \rightarrow 0 .
$$

In the following result, which is the core of the entire paper, we construct the sheaf $\mathcal{F}^{\Sigma}$ by using projective space-bundle arguments.

Theorem 3.4. Let $X$ be a smooth projective threefold. Let $\mathcal{F}$ be a globally generated rank-two vector bundle on $X$ and let $\delta$ be a positive integer. As in (2.9), let

$$
\begin{aligned}
\mathcal{V}_{\delta}(\mathcal{F})=\left\{[s] \in \mathbb{P}\left(H^{0}(\mathcal{F})\right) \mid C_{s}:=V(s) \subset X\right. \text { is irreducible } \\
\\
\text { with only } \delta \text { nodes as singularities }\} .
\end{aligned}
$$

Fix $[s] \in \mathcal{V}_{\delta}(\mathcal{F})$ and let $C=V(s) \subset X$. Denote by $\Sigma$ the set of nodes of $C$. Let

$$
\mathcal{P}:=\mathbb{P}_{X}(\mathcal{F}) \stackrel{\pi}{\longrightarrow} X
$$

be the projective space bundle together with its natural projection $\pi$ on $X$, and denote by $\mathcal{O}_{\mathcal{P}}(1)$ its tautological line bundle. Let

$$
\Sigma^{1}:=\mathbb{P}_{X}\left(T_{C}^{1}\right) \subset \mathcal{P}
$$

denote the zero-dimensional subscheme of $\mathcal{P}$ of length $\delta$, determined by the surjection (3.3). Denote by $\mathcal{I}_{\Sigma^{1 / \mathcal{P}}}$ the ideal sheaf of $\Sigma^{1}$ in $\mathcal{P}$. Then

(i) $\Sigma^{1}$ is a set of $\delta$ rational double points for the divisor $D_{s} \in\left|\mathcal{O}_{\mathcal{P}}(1)\right|$, corresponding to the given section $s \in H^{0}(X, \mathcal{F})$, and 
(ii) the subsheaf of $\mathcal{F}$ defined by

$$
\mathcal{F}^{\Sigma}:=\pi_{*}\left(\mathcal{I}_{\Sigma^{1} / \mathcal{P}} \otimes \mathcal{O}_{\mathcal{P}}(1)\right)
$$

is such that its global sections (modulo the one-dimensional subspace $\langle s\rangle$ ) parametrize first-order deformations of $s \in H^{0}(X, \mathcal{F})$ which are equisingular.

In particular, we have

$$
\frac{H^{0}\left(X, \mathcal{F}^{\Sigma}\right)}{\langle s\rangle} \cong T_{[s]}\left(\mathcal{V}_{\delta}(\mathcal{F})\right) \subset T_{[s]}\left(\mathbb{P}\left(H^{0}(\mathcal{F})\right)\right) \cong \frac{H^{0}(X, \mathcal{F})}{\langle s\rangle}
$$

Proof. To naturally define the sheaf $\mathcal{F}^{\Sigma}$ and the diagram (3.2), we consider the smooth, projective fourfold

$$
\mathcal{P}:=\mathbb{P}_{X}(\mathcal{F}) \stackrel{\pi}{\longrightarrow} X
$$

together with its tautological line bundle $\mathcal{O}_{\mathcal{P}}(1)$ such that $\pi_{*}\left(\mathcal{O}_{\mathcal{P}}(1)\right) \cong \mathcal{F}$. From

$$
0 \rightarrow \mathcal{O}_{X} \stackrel{\cdot s}{\rightarrow} \mathcal{F},
$$

we also have

$$
0 \rightarrow \mathcal{O}_{\mathcal{P}} \stackrel{\cdot s}{\rightarrow} \mathcal{O}_{\mathcal{P}}(1)
$$

Therefore, the nodal curve $C \subset X$ corresponds to a divisor $D_{s} \in\left|\mathcal{O}_{\mathcal{P}}(1)\right|$ on the fourfold $\mathcal{P}$. Take also

$$
\mathbb{F}:=\mathbb{P}_{C}^{1}=\mathcal{P} \operatorname{roj}\left(\mathcal{O}_{C}\left[\xi_{0}, \xi_{1}\right]\right) \stackrel{\pi_{1}}{\rightarrow} C,
$$

which is a ruled surface in $\mathcal{P}$. We want to study some geometric properties of $D_{s}$ and of $\mathbb{F}$. Let $p \in \Sigma=\operatorname{Sing}(C)$. Take an affine open set $U_{p} \subset X$ containing $p$, where the vector bundle $\mathcal{F}$ trivializes. Choose local coordinates $\underline{x}=\left(x_{1}, x_{2}, x_{3}\right)$ on $U_{p} \cong \mathbb{C}^{3}$ such that $\underline{x}(p)=(0,0,0)$ and such that the global section $s$ is

$$
\left.s\right|_{U_{p}}=\left(x_{1} x_{2}, x_{3}\right) .
$$

Then

$$
\mathcal{O}_{C}\left(U_{p}\right) \cong \mathbb{C}\left[x_{1}, x_{2}, x_{3}\right] /\left(x_{1} x_{2}, x_{3}\right),
$$

and

$$
\mathcal{O}_{\mathbb{F}}\left(\pi_{1}^{-1}\left(U_{p}\right)\right) \cong \mathbb{C}\left[x_{1}, x_{2}, x_{3}, \frac{\xi_{1}}{\xi_{0}}\right] /\left(x_{1} x_{2}, x_{3}\right) .
$$

Therefore, the surface $\mathbb{F}$ is singular along the lines in $\mathcal{L}=\bigcup_{i=1}^{\delta} L_{i}=\pi_{1}^{-1}(\Sigma)=$ $\pi^{-1}(\Sigma)$. As for $D_{s} \in\left|\mathcal{O}_{\mathcal{P}}(1)\right|$, since $U_{p}$ trivializes $\mathcal{F}$, then $\left.\mathcal{P}\right|_{U_{p}} \cong U_{p} \times \mathbb{P}^{1}$. Taking homogeneous coordinates $[u, v] \in \mathbb{P}^{1}$, we have $\mathcal{O}_{\mathcal{P}}\left(U_{p}\right) \cong \mathbb{C}\left[x_{1}, x_{2}, x_{3}, u, v\right]$. Thus,

$$
\mathcal{O}_{D_{s}}\left(U_{p}\right) \cong \mathbb{C}\left[x_{1}, x_{2}, x_{3}, u, v\right] /\left(v x_{3}+u x_{1} x_{2}\right) .
$$

By standard computations, we see that $D_{s}$ has a rational double point along the line $\pi^{-1}(p)=\pi_{1}^{-1}(p)$ which belongs to the singular locus of $\mathbb{F} \subset \mathcal{P}$.

Globally speaking, by using (3.3), we can state that the divisor $D_{s} \subset \mathcal{P}$ is singular along the locus

$$
\Sigma^{1}:=\mathbb{P}_{X}\left(T_{C}^{1}\right) \subset \mathcal{P}=\mathbb{P}_{X}(\mathcal{F}),
$$

where $\Sigma^{1} \cong \Sigma$ is a set of $\delta$ rational double points for $D_{s}$, each line of $\mathcal{L}=\pi^{-1}(\Sigma)$ containing only one of these points. Since $\Sigma^{1} \subset \mathcal{P}$ is a closed immersion, we have the exact sequence

$$
0 \rightarrow \mathcal{I}_{\Sigma^{1} / \mathcal{P}} \otimes \mathcal{O}_{\mathcal{P}}(1) \rightarrow \mathcal{O}_{\mathcal{P}}(1) \rightarrow \mathcal{O}_{\Sigma^{1}} \rightarrow 0
$$


which is defined by restricting $\mathcal{O}_{\mathcal{P}}(1)$ to $\Sigma^{1}$. By the definition of tautological line bundle, we have

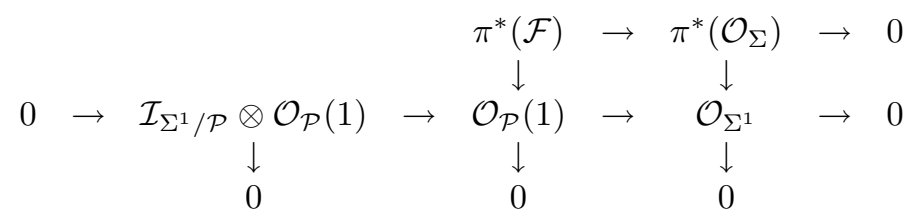

Since $\pi_{*}\left(\mathcal{O}_{\mathcal{P}}(1)\right) \cong \mathcal{F}$, it follows that

$$
\pi_{*}\left(\mathcal{O}_{\Sigma^{1}}\right)=\pi_{*}\left(\mathcal{O}_{\pi^{-1}\left(\Sigma^{1}\right)}\right)=\pi_{*}\left(\pi^{*}\left(\mathcal{O}_{\Sigma}\right)\right) \cong \mathcal{O}_{\Sigma}
$$

and since we have $\mathcal{F} \rightarrow \mathcal{O}_{\Sigma}$, by applying $\pi_{*}$ to the exact sequence (3.7), we get $\mathcal{R}^{1} \pi_{*}\left(\mathcal{I}_{\Sigma^{1} / \mathcal{P}} \otimes \mathcal{O}_{\mathcal{P}}(1)\right)=0$. Thus, we define

$$
\mathcal{F}^{\Sigma}:=\pi_{*}\left(\mathcal{I}_{\Sigma^{1} / \mathcal{P}} \otimes \mathcal{O}_{\mathcal{P}}(1)\right),
$$

which gives (3.5), so that

$$
0 \rightarrow \mathcal{F}^{\Sigma} \rightarrow \mathcal{F} \rightarrow \mathcal{O}_{\Sigma} \rightarrow 0
$$

as well as diagram (3.2), holds.

We remark that (3.6) gives a completely general characterization of the tangent space $T_{[s]}\left(\mathcal{V}_{\delta}(\mathcal{F})\right)$ on $X$. Furthermore, we have:

Corollary 3.9. With assumptions as in Theorem 3.4, from (3.8) we get

$$
\begin{aligned}
{[s] \in \mathcal{V}_{\delta}(\mathcal{F}) \text { is regular } } & \Leftrightarrow H^{0}(X, \mathcal{F}) \stackrel{\alpha_{X}}{\rightarrow} H^{0}\left(X, \mathcal{O}_{\Sigma}\right) \\
& \Leftrightarrow H^{0}\left(\mathcal{P}, \mathcal{O}_{\mathcal{P}}(1)\right) \stackrel{\alpha_{\mathcal{P}}}{\rightarrow} H^{0}\left(\mathcal{P}, \mathcal{O}_{\Sigma^{1}}\right) .
\end{aligned}
$$

Proof. This follows from Proposition 2.10 and from Theorem 3.4.

Note that, on the one hand, the map $\alpha_{X}$ in (3.10) is not defined by restricting the global sections of $\mathcal{F}$ to $\Sigma$, because (3.8) (i.e. the second row of diagram (3.2)) does not coincide with the restriction sequence

$$
\left.0 \rightarrow \mathcal{I}_{\Sigma / X} \otimes \mathcal{F} \rightarrow \mathcal{F} \rightarrow \mathcal{F}\right|_{\Sigma} \rightarrow 0
$$

precisely, we have

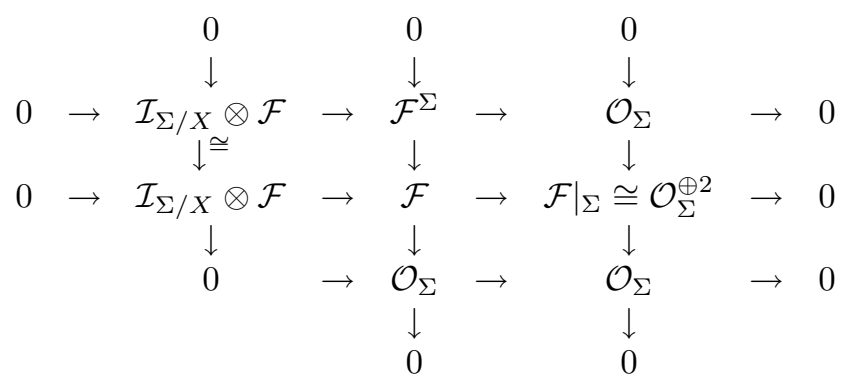

On the other hand, the exact sequence (3.7) on the fourfold $\mathcal{P}$ is equivalent to (3.8), by the Leray isomorphisms, but it is more naturally defined by restricting the line bundle $\mathcal{O}_{\mathcal{P}}(1)$ to $\Sigma^{1}$. Therefore, the map $\alpha_{\mathcal{P}}$ in (3.10) is a classical restriction map.

To better understand the map $\alpha_{X}$, we also want to give a local description of (3.8). 
Local description. Let $p \in \operatorname{Sing}(C)=\Sigma$ and take, as before, an affine open set

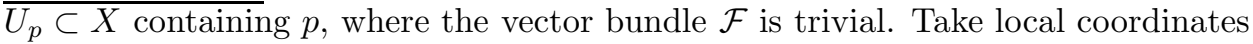
$\underline{x}=\left(x_{1}, x_{2}, x_{3}\right)$ on $U_{p} \cong \mathbb{C}^{3}$ such that $\underline{x}(p)=(0,0,0)$ and such that the global section $s$, whose zero-locus is $C$, is $\left.s\right|_{U_{p}}=\left(x_{1} x_{2}, x_{3}\right)$. Since $C=V\left(x_{1} x_{2}, x_{3}\right) \subset$ $\operatorname{Spec}\left(\mathbb{C}\left[x_{1}, x_{2}, x_{3}\right]\right) \cong U_{p}$, around the node $\underline{x}(p)=\underline{0}$ the map

$$
\left.\mathcal{T}_{\mathbb{C}^{3}}\right|_{C} \stackrel{J(s)}{\longrightarrow} \mathcal{N}_{C / \mathbb{C}^{3}} \rightarrow T_{C}^{1}
$$

is given by

$$
J(s):=\left(\begin{array}{lll}
\frac{\partial f_{1}}{\partial x_{1}} & \frac{\partial f_{1}}{\partial x_{2}} & \frac{\partial f_{1}}{\partial x_{3}} \\
\frac{\partial f_{2}}{\partial x_{1}} & \frac{\partial f_{2}}{\partial x_{2}} & \frac{\partial f_{2}}{\partial x_{3}}
\end{array}\right)=\left(\begin{array}{ccc}
x_{2} & x_{1} & 0 \\
0 & 0 & 1
\end{array}\right) .
$$

From the fact that $\operatorname{rank}\left(\left.J(s)\right|_{\underline{0}}\right)=1$, it follows that $\operatorname{coker}\left(\left.J(s)\right|_{\underline{0}}\right) \cong \mathbb{C}$. Let $s\left(x_{1}, x_{2}, x_{3}\right)=\left.s\right|_{U_{p}}=\left(x_{1} x_{2}, x_{3}\right)$; if $\sigma\left(x_{1}, x_{2}, x_{3}\right)$ is a section of $\mathcal{F}^{\Sigma}$ over $U_{p}$ then, by definition,

$$
s_{\epsilon}\left(x_{1}, x_{2}, x_{3}\right):=s\left(x_{1}, x_{2}, x_{3}\right)+\epsilon \sigma\left(x_{1}, x_{2}, x_{3}\right)
$$

is a first-order deformation of $s$ which determines equisingular zero-loci. Then

$$
\sigma\left(x_{1}, x_{2}, x_{3}\right)=J(s) \underline{u},
$$

where $\underline{u}=\underline{u}\left(x_{1}, x_{2}, x_{3}\right)=\left(u_{1}\left(x_{1}, x_{2}, x_{3}\right), u_{2}\left(x_{1}, x_{2}, x_{3}\right), u_{3}\left(x_{1}, x_{2}, x_{3}\right)\right)$. To see this, consider

$$
\begin{aligned}
\left.s(\underline{x}+\epsilon \underline{u})\right|_{\underline{0}} & \left.\equiv(s(\underline{x})+\epsilon J(s) \underline{u})\right|_{\underline{0}} \quad\left(\bmod \epsilon^{2}\right) \\
& =\left(\begin{array}{c}
x_{1} x_{2} \\
x_{3}
\end{array}\right)+\epsilon\left(\begin{array}{c}
x_{2} u_{1}+x_{1} u_{2} \\
u_{3}
\end{array}\right) \quad\left(\bmod \epsilon^{2}\right) ;
\end{aligned}
$$

thus

$$
s_{\epsilon}(\underline{x}) \equiv s(\underline{x}+\epsilon \underline{u}) \quad\left(\bmod \epsilon^{2}\right) .
$$

Moreover, since $U_{p}$ is a trivializing open subset for $\mathcal{F},(* *)$ becomes

$$
\begin{aligned}
& \mathcal{O}_{C}^{\oplus 3} \stackrel{J(s)}{\longrightarrow} \quad \mathcal{O}_{C}^{\oplus 2} \quad \rightarrow T_{C}^{1}, \\
& \left(e_{1}, e_{2}, e_{3}\right) \rightarrow\left(e_{1}^{\prime}, e_{2}^{\prime}\right) .
\end{aligned}
$$

Since $\operatorname{Im}(J(s))=\left\langle x_{2} e_{1}^{\prime}, x_{1} e_{1}^{\prime}, e_{2}^{\prime}\right\rangle$, then $e_{2}^{\prime}$ goes to zero in $T_{C}^{1}$, so the deformations in $(* * *)$ are actually equisingular.

Observe that, by (3.10) and some results in [10, one can immediately determine conditions for the regularity of $[s] \in \mathcal{V}_{\delta}(\mathcal{F})$. Indeed, if $\mathcal{F}$ is a globally generated rank-two vector bundle on $X$ which generates the 0 -jets at $\Sigma=\left\{p_{1}, \ldots, p_{\delta}\right\}$ (equiv. which separates the points of $\Sigma$ ), then by definition we have

$$
H^{0}(X, \mathcal{F}) \rightarrow \bigoplus_{i=1}^{\delta} \mathbb{C}_{(i)}^{2} .
$$

This implies the regularity conditions (3.10), as immediately follows by considering the last two columns of (3.11). In such a case an element $[s] \in \mathcal{V}_{\delta}(\mathcal{F})$ with $C=V(s)$ and $\Sigma=\operatorname{Sing}(C)$ is therefore a regular point. From Proposition 3.1 in [10], we deduce

Proposition 3.12. Let $X$ be a smooth, projective threefold. Let $\mathcal{G}$ be a nef ranktwo vector bundle and $L$ be a big and nef line bundle on $X$. Consider the rank-two vector bundle

$$
\mathcal{F}:=\mathcal{G} \otimes \omega_{X} \otimes \operatorname{det}(\mathcal{G}) \otimes \mathcal{O}_{X}(L)
$$


whose general section is assumed to be a smooth curve in $X$. Take $\delta$ to be a positive integer, and consider $[s] \in \mathcal{V}_{\delta}(\mathcal{F})$. Let $\Sigma=\left\{p_{1}, \ldots, p_{\delta}\right\}$ be the set of nodes of $C=V(s)$. Let $\epsilon(L, p)$ denote the Seshadri constant of the line bundle $L$ at the point $p$ (for the precise definition, see Remark 3.13 or Definition 5.3). Assume either

$$
\epsilon\left(L, p_{j}\right)>3 \delta, \forall p_{j} \in \Sigma,
$$

or

$$
L^{3}>\left(\epsilon\left(L, p_{j}\right)\right)^{3} \text { and } \epsilon\left(L, p_{j}\right) \geq 3 \delta, \forall p_{j} \in \Sigma .
$$

Then, the global sections of $\mathcal{F}$ separate $\Sigma$. In particular, $\mathcal{V}_{\delta}(\mathcal{F})$ is regular at $[s]$.

Proof. One applies the effective non-vanishing Theorem 2.2 in [10, taking $L_{j}=$ $\frac{1}{\delta} L$.

Remark 3.13. Take, e.g., $X \subset \mathbb{P}^{r}$, a smooth threefold, whose hyperplane section is denoted by $H$. Consider the line bundle $\mathcal{O}_{X}(k H)$, where $k$ is a positive integer, and take $\Sigma=\left\{p_{1}, \ldots, p_{\delta}\right\} \subset X$. Denote by $\mu_{j}$ the blowing-up of $X$ at the point $p_{j}$. Then, by definition,

$$
\epsilon\left(\mathcal{O}_{X}(k H), p_{j}\right):=\operatorname{Sup}\left\{\epsilon \in \mathbb{R}_{\geq 0} \mid \mu_{j}^{*}(k H)-\epsilon E_{j} \text { is a nef } \mathbb{R} \text {-divisor on } B l_{p_{j}}(X)\right\},
$$

where $E_{j}$ denotes the $\mu_{j}$-exceptional divisor. Equivalently,

$$
\epsilon\left(\mathcal{O}_{X}(k H), p_{j}\right):=\underset{\Gamma \subset X}{\operatorname{Inf}}\left\{\frac{k H \cdot \Gamma}{\text { mult }_{p_{j}}(\Gamma)}\right\}
$$

where the infimum is taken over all reduced and irreducible curves $\Gamma \subset X$ passing through $p_{j}$. Since $H$ is very ample on $X$, then the curve $\Gamma$, as a curve in $\mathbb{P}^{r}$, is such that $\operatorname{deg}(\Gamma) \geq \operatorname{mult}_{q}(\Gamma)$, for each $q \in \Gamma$. Therefore, the numerical conditions in Proposition 3.12 give

$$
\delta \leq \frac{k}{3}
$$

which is a linear bound on the admissible number of nodes of $C=V(s)$ in order for $[s] \in \mathcal{V}_{\delta}\left(\mathcal{G} \otimes \omega_{X} \otimes \operatorname{det}(\mathcal{G}) \otimes \mathcal{O}_{X}(k H)\right)$ to be a regular point.

However, the conditions on Seshadri constants are of local nature, and the results that one can deduce are strictly related to the postulation of the chosen points. In the next section, we shall discuss one of our results, which determines conditions on the vector bundle $\mathcal{F}$ and a uniform upper bound on the number of nodes $\delta$ such that each point of the scheme $\mathcal{V}_{\delta}(\mathcal{F})$ is regular.

\section{Some UNIFORM REGULARITY RESUlTS FOR $\mathcal{V}_{\delta}(\mathcal{E}(k))$}

From now on, let $X \subset \mathbb{P}^{r}$ be a smooth projective threefold with hyperplane bundle $\mathcal{O}_{X}(1)$, let $\mathcal{E}$ be a globally generated rank-two vector bundle on $X$, and let $k \geq 0, \delta>0$ be integers. With notation as in Section 3, we shall always take

$$
\mathcal{F}=\mathcal{E} \otimes \mathcal{O}_{X}(k)=\mathcal{E}(k)
$$

and consider the scheme $\mathcal{V}_{\delta}(\mathcal{E}(k))$ on $X$. By using Theorem 3.4 and Corollary 3.9, here we determine conditions on the vector bundle $\mathcal{E}$ and on the integer $k$ and uniform upper bounds on the number of nodes $\delta$ implying that each point of $\mathcal{V}_{\delta}(\mathcal{E}(k))$ is regular. First we need the following result. 
Proposition 4.1. Let $X \subset \mathbb{P}^{r}$ be a smooth threefold with hyperplane bundle $\mathcal{O}_{X}(1)$. Let $\mathcal{E}$ be a globally generated rank-two vector bundle on $X$, and take $k>0$ such that $\mathcal{O}_{X}(k)$ separates $\delta$ distinct given points $\Sigma=\left\{p_{1}, \ldots, p_{\delta}\right\}$, i.e. the restriction map

$$
H^{0}\left(X, \mathcal{O}_{X}(k)\right) \stackrel{\rho_{k}}{\rightarrow} H^{0}\left(\mathcal{O}_{\Sigma}\right)
$$

is surjective. If $[s] \in \mathcal{V}_{\delta}(\mathcal{E}(k))$ determines a nodal curve $C$ in $X$ with $\operatorname{Sing}(C)=\Sigma$, then $[s] \in \mathcal{V}_{\delta}(\mathcal{E}(k))$ is a regular point.

Proof. Since $\mathcal{E}$ is globally generated on $X$, the evaluation morphism

$$
H^{0}(X, \mathcal{E}) \otimes \mathcal{O}_{X} \stackrel{e v}{\rightarrow} \mathcal{E}
$$

is surjective. This means that, for each $p \in X$, there exist global sections $s_{1}^{(p)}, s_{2}^{(p)} \in$ $H^{0}(X, \mathcal{E})$ such that

$$
s_{1}^{(p)}(p)=(1,0), s_{2}^{(p)}(p)=(0,1) \in \mathcal{O}_{X, p}^{\oplus 2} .
$$

Condition (4.2) means there exist global sections $\sigma_{1}, \ldots, \sigma_{\delta} \in H^{0}\left(X, \mathcal{O}_{X}(k)\right)$ such that

$$
\sigma_{i}\left(p_{j}\right)=\underline{0} \in \mathbb{C}^{\delta} \text { if } i \neq j, \quad \text { and } \quad \sigma_{i}\left(p_{i}\right)=(0, \ldots, \stackrel{i-t h}{1}, \ldots, 0), 1 \leq i \leq \delta .
$$

Therefore, from our hypotheses, it immediately follows that

$$
H^{0}(X, \mathcal{E}(k)) \rightarrow H^{0}\left(\mathcal{O}_{\Sigma}^{\oplus 2}\right) \cong \mathbb{C}^{2 \delta} .
$$

If we take $\mathcal{P}=\mathbb{P}_{X}(\mathcal{E}(k)) \stackrel{\pi}{\longrightarrow} X$ and if we consider, as in $(3.5),(\mathcal{E}(k))^{\Sigma}:=$ $\pi_{*}\left(\mathcal{I}_{\Sigma^{1} / \mathcal{P}} \otimes \mathcal{O}_{\mathcal{P}}(1)\right)$, from diagram (3.11), we get:

$$
\begin{array}{rll}
H^{0}(\mathcal{E}(k)) & \rightarrow & H^{0}\left(\mathcal{O}_{\Sigma}^{\oplus 2}\right) \cong \mathbb{C}^{2 \delta} \\
\downarrow^{\mu} & & \downarrow \\
H^{0}\left(\mathcal{O}_{\Sigma}\right) & \cong & H^{0}\left(\mathcal{O}_{\Sigma}\right) \cong \mathbb{C}^{\delta} \\
& & \downarrow \\
& & 0
\end{array}
$$

Thus $\mu$ is surjective. By (3.10) and the local description of $\alpha_{X}$ we get the desired conclusion.

Remark 4.3. With the previous result, the regularity condition (3.10) translates into the surjectivity of the restriction map $\rho_{k}$ in (4.2), which is a natural restriction map of line bundles on the threefold $X$.

The following more general proposition gives an effective and uniform bound on the number $\delta=|\Sigma|$, in terms of the integer $k$, in order for the map $\rho_{k}$ to be surjective.

Proposition 4.4. Let $X$ be a smooth projective $m$-fold, $L$ a very ample line bundle and $k$ a positive integer. Then, $L^{\otimes k}$ separates any set $\Sigma$ of $\delta$ distinct point of $X$ with $\delta \leq k+1$. In particular, the map $\rho_{k}$ in (4.2) is surjective, for each such $\Sigma \subset X$.

Proof. Since $L$ is very ample on $X$, for every $p_{1} \neq p_{2} \in X$, there exists a section $s_{1,2} \in H^{0}(X, L)$ such that

$$
s_{1,2}\left(p_{1}\right)=1 \text { and } s_{1,2}\left(p_{2}\right)=0 .
$$


If $p_{3} \in X$ is such that $p_{3} \neq p_{1}, p_{2}$, there exists $s_{1,3} \in H^{0}(X, L)$ such that

$$
s_{1,3}\left(p_{1}\right)=1 \text { and } s_{1,3}\left(p_{3}\right)=0 .
$$

Then

is such that

$$
\sigma:=s_{1,2} \otimes s_{1,3} \in H^{0}\left(X, L^{\otimes 2}\right)
$$

$$
\sigma\left(p_{1}\right)=1, \sigma\left(p_{2}\right)=0, \sigma\left(p_{3}\right)=0 .
$$

With analogous computations, it follows that $L^{\otimes 2}$ separates three points of $X$. Recursively, $L^{\otimes k}$ separates $k+1$ distinct points in $X$.

Finally, we have the main result of this section.

Theorem 4.5. Let $X \subset \mathbb{P}^{r}$ be a smooth threefold with hyperplane bundle $\mathcal{O}_{X}(1)$. Let $\mathcal{E}$ be a globally generated rank-two vector bundle on $X$, and let $k \geq 0$ and $\delta>0$ be integers. If

$$
\delta \leq k+1
$$

then $\mathcal{V}_{\delta}(\mathcal{E}(k)$ is regular at each point.

Proof. If $k=0$, then $\delta=1$; therefore, by the hypothesis on $\mathcal{E}$, it follows that

$$
H^{0}(\mathcal{E}) \rightarrow H^{0}\left(\mathcal{O}_{p}^{\oplus 2}\right)
$$

is surjective, for each $p \in X$. This implies that $\mathcal{V}_{1}(\mathcal{E})$ is regular at each point.

When $k>0$, the statement follows from Theorem 3.4, Propositions 4.1, 4.4 and Remark 4.3

Remark 4.7. Observe that the bound (4.6) is uniform, i.e. it does not depend on the postulation of nodes of the curves which are zero-loci of sections parametrized by $\mathcal{V}_{\delta}\left(\mathcal{E} \otimes L^{\otimes k}\right)$. We remark that Theorem 4.5 generalizes what Ballico and Chiantini proved in [1], mainly because, by the characterization given in our Theorem 3.4 ] our approach more generally holds for families of nodal curves on smooth projective threefolds, but also because, even in the case $X=\mathbb{P}^{3}$, the main subject of [1, our result is effective, and not asymptotic like Proposition 3.1 in [1]. Furthermore, Ballico and Chiantini showed that in the asymptotic case, i.e. with $k>>0$, the bound $\delta \leq k+1$ is almost sharp. Indeed, they constructed an example of a nonregular point $[s] \in \mathcal{V}_{k+4}\left(\mathcal{O}_{\mathbb{P}^{3}}(k+1) \oplus \mathcal{O}_{\mathbb{P}^{3}}(k+4)\right)$ whose corresponding curve $C$ has its $(k+4)$ nodes lying on a line $L \subset \mathbb{P}^{3}$; they also showed that, when the points are moved so that they are no longer aligned, then $[s]$ is regular. Thanks to Theorem 4.5, the same example works not only in the asymptotic case but for each $k \geq 3$, proving the almost sharpness of the bound (4.6).

In the next section, we also discuss some other examples of nodal curves on smooth projective threefolds which determine non-regular points of some $\mathcal{V}_{\delta}(\mathcal{E}(k))$ (see Remarks 5.21 and 5.38).

\section{Regularity Results via Seshadri constants AND POSTULATION OF NODES}

As already observed in the previous section, given a smooth threefold $X \subset \mathbb{P}^{r}$, a globally generated rank-two vector bundle $\mathcal{E}$ on $X$ and integers $\delta>0, k \geq 0$, Theorem 4.5 determines sufficient conditions for each point of the scheme $\mathcal{V}_{\delta}(\mathcal{E}(k))$ to be regular, for every non-negative integer $k$. Using a local analysis, we can 
determine some other regularity results which take into account the postulation of nodes of the curves related to the elements parametrized by $\mathcal{V}_{\delta}(\mathcal{E}(k))$. Precisely, let $[s] \in \mathcal{V}_{\delta}(\mathcal{E}(k)), C=V(s)$, and denote by $\Sigma=\operatorname{Sing}(C)$ its set of nodes. Our aim is to find conditions on $\Sigma$ which determine finer estimates on the admissible number $\delta$ of nodes in order to get the regularity of the point $[s] \in \mathcal{V}_{\delta}(\mathcal{E}(k))$.

Remark 5.1. By Proposition 4.1 and Remark 4.3, a sufficient condition for the regularity of $[s] \in \mathcal{V}_{\delta}(\mathcal{E}(k))$ is

$$
h^{1}\left(X, \mathcal{I}_{\Sigma / X} \otimes \mathcal{O}_{X}(k)\right)=0 .
$$

Observe that, if $\omega_{X}^{\vee} \otimes \mathcal{O}_{X}(k)$ is a big and nef line bundle on $X$, then, by the Kawamata-Viehweg vanishing theorem, (5.2) is exactly equivalent to surjectivity of $\rho_{k}$ in (4.2) which implies the regularity of $[s] \in \mathcal{V}_{\delta}(\mathcal{E}(k))$. In the sequel we will be concerned with finding sufficient conditions implying (5.2); we shall focus on the case when $X$ is a Fano or a Calabi-Yau threefold and, in particular, when $X=\mathbb{P}^{3}$.

First of all, we have to recall the following general definitions from [11, 12] and 24].

Definition 5.3. Let $L$ be a nef line bundle on an $n$-dimensional projective variety $Y$. Let $p \in Y$ and let $b_{1}: Y_{1} \rightarrow Y$ denote the blowing-up of $Y$ at $p$. The Seshadri constant of $L$ at $p, \epsilon(L, p)$, is defined as

$$
\epsilon(L, p):=\operatorname{Sup}\left\{\epsilon \in \mathbb{R}_{\geq 0} \mid b_{1}^{*}(L)-\epsilon E \text { is a nef } \mathbb{R} \text {-divisor on } Y_{1}\right\},
$$

where $E$ denotes the $b_{1}$-exceptional divisor. Equivalently,

$$
\epsilon(L, p):=\underset{\Gamma \subset Y}{\operatorname{Inf}}\left\{\frac{L \cdot \Gamma}{\operatorname{mult}_{p}(\Gamma)}\right\},
$$

where the infimum is taken over all reduced and irreducible curves $\Gamma \subset Y$ passing through $p$.

More generally, if $\delta$ is an integer greater than 1 and $p_{1}, \ldots, p_{\delta} \in Y$ are $\delta$ distinct points, then, denoting by $b_{\delta}: Y_{\delta} \rightarrow Y$ the blowing-up of $Y$ along the given points, the multiple point Seshadri constant at $p_{1}, \ldots, p_{\delta}$ is defined as

$$
\epsilon\left(L, p_{1}, \ldots, p_{\delta}\right):=\operatorname{Sup}\left\{\epsilon \in \mathbb{R}_{\geq 0} \mid b_{\delta}^{*}(L)-\epsilon \sum_{i=1}^{\delta} E_{i} \text { is a nef } \mathbb{R} \text {-divisor on } Y_{\delta}\right\}
$$

where $\sum_{i=1}^{\delta} E_{i}$ is the $b_{\delta}$-exceptional divisor. As before, one also has

$$
\epsilon\left(L, p_{1}, \ldots, p_{\delta}\right):=\operatorname{Inf}_{\Gamma \subset Y}\left\{\frac{L \cdot \Gamma}{\sum_{i=1}^{\delta} \operatorname{mult}_{p_{i}}(\Gamma)}\right\}
$$

where the infimum is taken over all integral curves $\Gamma \subset Y$ with $\Gamma \cap\left\{p_{1}, \ldots, p_{\delta}\right\} \neq \emptyset$.

Definition 5.8. Let $Y$ be a projective variety of dimension $n$, and let $\delta \geq 2$ be a positive integer. Let $Y^{(\delta)}$ denote the $\delta$-Cartesian product of $Y$ minus the diagonals. If $\left(p_{1}, \ldots, p_{\delta}\right) \in Y^{(\delta)}$, the points $p_{1}, \ldots, p_{\delta}$ are called general points of $Y$ if $\left(p_{1}, \ldots, p_{\delta}\right)$ is outside a Zarisky closed subset of $Y^{(\delta)}$, and very general points of $Y$ if $\left(p_{1}, \ldots, p_{\delta}\right)$ is outside the union of countably many proper subvarieties of $Y^{(\delta)}$.

Before stating our next result, we recall that from our assumptions in $\S 2$ (see Definition 2.11 and above) the integer $\delta$ is always assumed to be $\delta \leq \min \left\{h^{0}(\mathcal{E}(k))-\right.$ $1, p_{a}(C)$. 
Theorem 5.9. Let $X \subset \mathbb{P}^{r}$ be a smooth threefold such that $\operatorname{deg}(X)=d, \omega_{X} \cong$ $\mathcal{O}_{X}(-m)$ for some integers $d>0$ and $m \geq 0$. Let $\mathcal{E}$ be a globally generated ranktwo vector bundle on $X$ and let $k \geq 0$ and $\delta>0$ be integers. Let $[s] \in \mathcal{V}_{\delta}(\mathcal{E}(k))$, $C=V(s)$, and let $\Sigma$ denote its set of nodes. Assume either

(i) $k+m>3$, when $\delta=1$, or

(ii) $\Sigma$ is a set of $\delta \geq 2$ very general points on $X$ and

a) $k+m>\frac{6}{\sqrt[3]{d}}$, when $d<8$ and $\delta \leq 5$;

b)

$$
k+m>\max \left\{\frac{36}{\operatorname{deg}(C)}, \frac{18}{\sqrt[3]{25 d}}\right\},
$$

when $d<8$ and $6 \leq \delta<\min \left\{h^{0}(\mathcal{E}(k)), \frac{1}{6}(k+m) \operatorname{deg}(C), \delta_{0}^{(k)}\right\}$, where $\delta_{0}^{(k)}$ is a root of the polynomial $F_{k, m, d}(\delta):=27 \delta^{3}-(k+m)^{3} d(\delta-1)^{2}$ such that $F_{k, m, d}(\delta)<0$ on the connected interval $\left[6, \delta_{0}^{(k)}\right)$;

c) $k+m>3$, when $d \geq 8$ and $\delta \leq d-2$;

d)

$$
k+m>\max \left\{\frac{6(d-2)}{\operatorname{deg}(C)}, \frac{3(d-2)}{\sqrt[3]{d(d-2)^{2}}}\right\}
$$

when $d \geq 8$ and $d-2 \leq \delta<\min \left\{h^{0}(\mathcal{E}(k)), \frac{1}{6}(k+m) \operatorname{deg}(C), \delta_{0}^{(k)}\right\}$, where $\delta_{0}^{(k)}$ is a root of the polynomial $F_{k, m, d}(\delta):=27 \delta^{3}-(k+m)^{3} d(\delta-1)^{2}$ such that $F_{k, m, d}(\delta)<0$ on the connected interval $\left[d-2, \delta_{0}^{(k)}\right)$.

Then, in each case, $[s]$ is a regular point of $\mathcal{V}_{\delta}(\mathcal{E}(k))$.

Proof. Let $b_{\delta}: Y_{\delta} \rightarrow X$ be the blowing-up of $X$ along $\Sigma$. From our assumptions on $X$ and from Leray's isomorphism, it follows that

$$
\begin{aligned}
H^{1}\left(X, \mathcal{I}_{\Sigma / X}(k)\right) & =H^{1}\left(X, \mathcal{I}_{\Sigma / X}(k+m) \otimes \omega_{X}\right) \\
& \cong H^{1}\left(Y_{\delta}, \omega_{Y_{\delta}} \otimes \mathcal{O}_{Y_{\delta}}\left((k+m) b_{\delta}^{*}(H)-3 B\right)\right),
\end{aligned}
$$

where $B=\sum_{i=1}^{\delta} E_{i}$ is the $b_{\delta}$-exceptional divisor. Therefore, if $(k+m) b_{\delta}^{*}(H)-3 B$ is a big and nef divisor, by the Kawamata-Viehweg vanishing theorem,

$$
H^{1}\left(X, \mathcal{I}_{\Sigma / X}(k)\right)=(0),
$$

which implies the regularity of $[s] \in \mathcal{V}_{\delta}(\mathcal{E}(k))$ (see Remark [5.1).

Let $\bar{\epsilon}=\epsilon\left(\mathcal{O}_{X}(1), \Sigma\right)$ denote the multiple point Seshadri constant of the very ample line bundle $\mathcal{O}_{X}(1)$ at $\Sigma$; then

$$
(k+m) b_{\delta}^{*}(H)-3 B=\frac{3}{\bar{\epsilon}}\left(b_{\delta}^{*}(H)-\bar{\epsilon} B\right)+\left(k+m-\frac{3}{\bar{\epsilon}}\right) b_{\delta}^{*}(H) .
$$

Observe that the first summand in the right-hand side is nef, by definition of $\bar{\epsilon}$, whereas the second is big and nef as soon as $\bar{\epsilon}>\frac{3}{k+m}$.

We want to show that our hypotheses imply that the Seshadri constant $\bar{\epsilon}$ is always greater than $\frac{3}{k+m}$; then the statement will be proved.

(i) If $\delta=1$, then $\bar{\epsilon}=\epsilon\left(\mathcal{O}_{X}(1), p\right) \geq 1$ for each $p \in X$, since $\mathcal{O}_{X}(1)$ is very ample. Therefore, since $k+m>3$ implies $\frac{3}{k+m}<1$, we have $h^{1}\left(X, \mathcal{I}_{\{p\} / X}(k)\right)=0$ for each $p \in X$.

(ii) For $\delta \geq 2$, we can consider Theorem 1.1 in [24]. For $L$ a big and nef line bundle on $X$, the author of [24] denotes by $\epsilon(L ; \delta)$ the Seshadri constant of $L$ at 
$\delta$ very general points of $X$, and by $\epsilon(L ; 1)$ the Seshadri constant of $L$ at a very general point of $X$. In the threefold case with $L=\mathcal{O}_{X}(1)$, Küchle's result gives

$$
\epsilon\left(\mathcal{O}_{X}(1) ; \delta\right) \geq M:=\min \left\{\epsilon\left(\mathcal{O}_{X}(1) ; 1\right), \frac{\sqrt[3]{d}}{2}, \frac{\sqrt[3]{d(\delta-1)^{2}}}{\delta}\right\},
$$

where $\epsilon\left(\mathcal{O}_{X}(1) ; 1\right) \geq 1$, since $\mathcal{O}_{X}(1)$ is very ample. By assumption, $\Sigma$ is a set of very general points on $X$; thus $\bar{\epsilon}=\epsilon\left(\mathcal{O}_{X}(1) ; \Sigma\right)$ coincides with $\epsilon\left(\mathcal{O}_{X}(1) ; \delta\right)$. Therefore, to prove that $\bar{\epsilon}>\frac{3}{k+m}$ we need only show that our numerical hypotheses imply

$$
\frac{3}{k+m}<M \text {. }
$$

Observe that, when $d \geq 8$ and $\delta \leq d-3$, we have $M \geq 1$, since all the real numbers in the brackets in (5.10) are greater than or equal to 1 . Since $k+m>3$, (5.11) trivially holds.

In the other cases, we find that:

- $\epsilon\left(\mathcal{O}_{X}(1) ; 1\right)$ is always greater than or equal to 1 , since $\mathcal{O}_{X}(1)$ is very ample;

- $\frac{\sqrt[3]{d}}{2}<1$ iff $d<8$

- $\frac{\sqrt[3]{d(\delta-1)^{2}}}{\delta}<1$ if $\delta \geq d-2$, when $d \geq 3$, or if $\delta \geq 2$, when $1 \leq d \leq 2$;

- $\frac{\sqrt[3]{d(\delta-1)^{2}}}{\delta}<\frac{\sqrt[3]{d}}{2}$ iff $\delta \geq 6$.

Therefore, considering all the above inequalities, we find that

$$
M=\left\{\begin{array}{lll}
\frac{\sqrt[3]{d}}{2} & \text { if } \quad d<8 \text { and } 2 \leq \delta \leq 5, \\
\frac{\sqrt[3]{d(\delta-1)^{2}}}{\delta} & \text { if } \quad \begin{array}{l}
\text { either } d \geq 8 \text { and } \delta \geq d-2 \\
\end{array} & \text { or } d<8 \text { and } \delta \geq 6 .
\end{array}\right.
$$

In all these cases we have $M<1$.

When $M=\frac{\sqrt[3]{d}}{2}$, (5.11) holds as soon as $k+m>\frac{6}{\sqrt[3]{d}}$. On the other hand, when $M=\frac{\sqrt[3]{d(\delta-1)^{2}}}{\delta}$, we want

$$
\frac{3}{k+m}<\frac{\sqrt[3]{d(\delta-1)^{2}}}{\delta}
$$

Since this case occurs when $d \geq 8, \delta \geq d-2$ and when $d<8, \delta \geq 6$, we impose

$$
\frac{3(d-2)}{\sqrt[3]{d(d-3)^{2}}}<k+m, \text { when } d \geq 8
$$

and

$$
\frac{18}{\sqrt[3]{25 d}}<k+m, \text { when } d<8 .
$$

Observe that (5.12) is equivalent to asking that the polynomials

$$
F_{k, m, d}(\delta):=27 \delta^{3}-d(k+m)^{3}(\delta-1)^{2}
$$

satisfy the inequalities $F_{k, m, d}(\delta)<0$. By (5.13) and (5.14), we have

$$
\begin{gathered}
F_{k, m, d}(d-2)<0, \text { when } d \geq 8, \\
F_{k, m, d}(6)<0, \text { when } d<8 .
\end{gathered}
$$


Therefore each cubic polynomial $F_{k, m, d}(\delta)$ has (at least) one root which is greater than $d-2$ when $d \geq 8$, and greater than 6 when $d<8$. Denote by $\delta_{0}^{(k)}$ the root of $F_{k, m, d}(\delta)$ such that

$$
\begin{gathered}
F_{k, m, d}(\delta)<0, \forall \delta \in\left[d-2, \delta_{0}^{(k)}\right), \quad \text { when } d \geq 8, \\
F_{k, m, d}(\delta)<0, \forall \delta \in\left[6, \delta_{0}^{(k)}\right), \quad \text { when } d<8 .
\end{gathered}
$$

Thus, in such ranges of values for $\delta$, (5.11) automatically holds.

On the other hand, since $[s] \in \mathcal{V}_{\delta}(\mathcal{E})$, then $C \subset X$ is an irreducible curve having nodes at $\Sigma$; thus, by definition of a multiple point Seshadri constant, see (5.7), we have $\frac{\operatorname{deg}(C)}{m u l t_{\Sigma}(C)}>\frac{3}{k+m}$, i.e. $\delta<\frac{1}{6}(k+m) \operatorname{deg}(C)$. Therefore, when $\delta \geq d-2$, we have $(k+m)>\frac{6(d-2)}{\operatorname{deg}(C)}$, whereas $\delta \geq 6$ gives $(k+m)>\frac{36}{\operatorname{deg}(C)}$.

When, in particular, $X=\mathbb{P}^{3}$, we can simplify the previous result.

Corollary 5.15. Let $\mathcal{E}$ be a globally generated rank-two vector bundle on $\mathbb{P}^{3}$. Denote by $c_{i}$ the $i^{\text {th }}$ Chern class of $\mathcal{E}$. Let $k$ and $\delta$ be integers such that $k \geq 0$ and $\delta>0$. Let $[s] \in \mathcal{V}_{\delta}(\mathcal{E}(k))$, and let $\Sigma$ denote the set of nodes of the curve $C \subset \mathbb{P}^{3}$ corresponding to $s$. Assume that

(i) $k \geq 0$, when $\delta=1$;

(ii) $k \geq 3$, when:

a) $\delta=2$, or

b) $3 \leq \delta \leq 5$ and $\Sigma$ is a set of very general points in $\mathbb{P}^{3}$, or

c) $3 c_{1}+c_{2}+4>0, \Sigma$ is a set of very general points in $\mathbb{P}^{3}$ and $6 \leq \delta<$ $\min \left\{h^{0}(\mathcal{E}(k)), \frac{1}{6}(k+4)\left(k^{2}+c_{1} k+c_{2}\right), \delta_{0}^{(k)}\right\}$, where $\delta_{0}^{(k)}$ is a positive root of the polynomial $F_{k},(\delta):=27 \delta^{3}-(k+4)^{3}(\delta-1)^{2}$ such that $F_{k}(\delta)<0$ on the connected interval $\left[6, \delta_{0}^{(k)}\right)$.

Then, in each case, $[s]$ is a regular point of $\mathcal{V}_{\delta}(\mathcal{E}(k))$.

Proof. As in the proof of Theorem [5.9, it suffices to show that the multiple point Seshadri constant $\bar{\epsilon}:=\epsilon\left(\mathcal{O}_{X}(1) ; \Sigma\right)>\frac{3}{k+4}$.

If $\delta=1, \bar{\epsilon}=\epsilon\left(\mathcal{O}_{X}(1) ; p\right)=1$ for each $p \in \mathbb{P}^{3}$, since there exist lines in $\mathbb{P}^{3}$. Thus, for each $k \geq 0$ we have $\bar{\epsilon}>\frac{3}{k+4}$.

If $\delta=2$, then $\bar{\epsilon}=\epsilon\left(\mathcal{O}_{X}(1) ; p_{1}, p_{2}\right) \geq \frac{1}{2}$ for $\left(p_{1}, p_{2}\right) \in\left(\mathbb{P}^{3}\right)^{(2)}$ (as in Definition [5.8), since for all $p_{1} \neq p_{2}$ there exists the line $L_{p_{1}, p_{2}}=<p_{1}, p_{2}>$. Therefore, if $k \geq 3, \bar{\epsilon}>\frac{3}{k+4}$.

For $\delta \geq 3$ we can use the same procedure of Theorem [5.9, observing that $\epsilon\left(\mathcal{O}_{X}(1) ; \Sigma\right)=\epsilon\left(\mathcal{O}_{X}(1) ; \delta\right)$, by assumption on $\Sigma$, and that $\epsilon\left(\mathcal{O}_{X}(1), \delta\right) \geq M:=$ $\min \left\{1, \frac{1}{2}, \frac{\sqrt[3]{(\delta-1)^{2}}}{\delta}\right\}$.

Remark 5.16. Observe that the polynomials $F_{k}(\delta)$ in Corollary 5.15 asymptotically give the upper bounds $\delta<\frac{(k+4)^{3}}{27}$ (equivalently, $k>3 \sqrt[3]{\delta}-4$ ). Therefore, we have a cubic polynomial in the indeterminate $k$ which bounds the admissible number of nodes of $C$. The same occurs with the inequalities $\delta<h^{0}(X, \mathcal{E}(k))$ and $\delta<$ $\frac{1}{6}(k+4)\left(k^{2}+c_{1} k+c_{2}\right)$. Similarly for the polynomials $F_{k, m, d}(\delta)$ in Theorem [5.9 Therefore, we have cubic upper bounds on $k$ for $\delta$ to get regularity results for the point $[s] \in \mathcal{V}_{\delta}(\mathcal{E}(k))$. This depends on the fact that the computations are related to Seshadri constants of very ample line bundles at very general points on a 3-dimensional variety. 
Remark 5.17. At this point, on the one hand we have Theorem 4.5, which gives uniform bounds on the admissible number $\delta$ of nodes in order for each point of the Severi variety $\mathcal{V}_{\delta}(\mathcal{E}(k))$ on a threefold $X$ to be regular; morover, these uniform upper bounds only depend on the number of nodes, and not on their configurations in $X$. On the other hand, Theorem 5.9 determines sufficient conditions for the regularity of a point $[s] \in \mathcal{V}_{\delta}(\mathcal{E}(k))$, having assumed that the nodes of $C=V(s)$ are in very general position on $X$. Therefore, there are intermidiate cases which are very interesting to study. Specifically, if $[s] \in \mathcal{V}_{\delta}(\mathcal{E}(k))$, we want to find conditions for its regularity, assuming that a (not necessarily proper) subset of the nodes of $C$ lies on a proper subscheme of $X$.

Given a smooth threefold $X \subset \mathbb{P}^{r}$, a globally generated rank-two vector bundle $\mathcal{E}$ on $X$, and positive integers $k$ and $\delta$, consider $[s] \in \mathcal{V}_{\delta}(\mathcal{E}(k))$. From now on, $C$ will denote the irreducible curve determined by $s$, whose set of nodes is $\Sigma=\operatorname{Sing}(C)$, and $\Sigma_{0} \subseteq \Sigma$ will denote a (not necessarily proper) subset of nodes of $C$.

Proposition 5.18. Let $X \subset \mathbb{P}^{r}$ be a smooth threefold and let $H$ denote its hyperplane section. Let $S_{a} \subset X$ be an irreducible divisor such that $S_{a} \sim a H$ on $X$, for some positive integer a. Let $[s] \in \mathcal{V}_{\delta}(\mathcal{E}(k)), C=V(s)$, and assume that $\Sigma_{0} \subset \Sigma=\operatorname{Sing}(C)$ lies on $S_{a} \backslash \operatorname{Sing}\left(S_{a}\right)$. Assume also that

$$
h^{1}\left(X, \mathcal{O}_{X}(k-a)\right)=h^{1}\left(X, \mathcal{O}_{X}(k)\right)=h^{2}\left(X, \mathcal{O}_{X}(k-a)\right)=0
$$

(e.g, when $X$ is arithmetically Cohen-Macaulay). Then, if $\Sigma_{0}$ does not impose independent conditions to the complete linear system in $\left|\mathcal{O}_{S_{a}}(k)\right|$ on $S_{a}$, [s] cannot be a regular point for $\mathcal{V}_{\delta}(\mathcal{E}(k))$.

Proof. Since $S_{a} \sim a H$ on $X$, we have the ideal sequence

$$
0 \rightarrow \mathcal{O}_{X}(k-a) \rightarrow \mathcal{I}_{\Sigma_{0} / X}(k) \rightarrow \mathcal{I}_{\Sigma_{0} / S_{a}}(k) \rightarrow 0
$$

Therefore, by the hypotheses on $X, H^{1}\left(X, \mathcal{I}_{\Sigma_{0} / X}(k)\right) \cong H^{1}\left(S_{a}, \mathcal{I}_{\Sigma_{0} / S_{a}}(k)\right)$, which implies the statement.

In particular,

Corollary 5.20. Let $S_{a} \subset \mathbb{P}^{3}$ be an irreducible (not necessarily smooth) surface of degree a. Let $[s] \in \mathcal{V}_{\delta}(\mathcal{E}(k)), C=V(s)$, and assume that $\Sigma_{0} \subseteq \Sigma=\operatorname{Sing}(C)$ is such that $\Sigma_{0} \subset S_{a} \backslash \operatorname{Sing}\left(S_{a}\right)$. Then, if $\Sigma_{0}$ does not impose independent conditions on the complete linear system $\left|\mathcal{O}_{S_{a}}(k)\right|$ on $S_{a},[s]$ is not a regular point for $\mathcal{V}_{\delta}(\mathcal{E}(k))$.

Remark 5.21. Observe that, with Proposition 5.18 and Corollary [5.20, one can easily construct many examples of non-regular points $[s] \in \mathcal{V}_{\delta}(\mathcal{E}(k))$, corresponding to nodal curves on a smooth projective threefold $X$, by translating the problem to linear systems on surfaces $S \subset X$ not separating a given set of smooth points in $S$.

On the other hand, one can also find conditions on $\delta_{0}=\left|\Sigma_{0}\right|$ ensuring that, if $[s] \in \mathcal{V}_{\delta}(\mathcal{E}(k))$ is not regular, the failure of the regularity property depends on the behaviour of the nodes in $\Sigma \backslash \Sigma_{0}$. Indeed, since $\Sigma_{0} \subseteq \Sigma$, we have

$$
0 \rightarrow \mathcal{I}_{\Sigma / X}(k) \rightarrow \mathcal{I}_{\Sigma_{0} / X}(k) \rightarrow \mathcal{O}_{\Sigma \backslash \Sigma_{0}}(k) \rightarrow 0
$$

Taking $X$ as in Proposition 5.18 and assuming that $h^{1}\left(S_{a}, \mathcal{O}_{S_{a}}(k)\right)=0$ (e.g, for $\left.X=\mathbb{P}^{3}\right)$, then if we have some conditions implying that $\left|\mathcal{O}_{S_{a}}(k)\right|$ separates $\Sigma_{0}$ on $S_{a}$, by (5.22) we have

$$
H^{0}\left(\mathcal{O}_{\Sigma \backslash \Sigma_{0}}(k)\right) \rightarrow H^{1}\left(\mathcal{I}_{\Sigma / X}(k)\right)
$$


Therefore, a possibly non-zero element in $H^{1}\left(X, \mathcal{I}_{\Sigma / X}(k)\right)$ is induced by an element in $H^{0}\left(\mathcal{O}_{\Sigma \backslash \Sigma_{0}}(k)\right)$.

To get some effective results, we can use several approaches. First of all, we want to consider the case when $S_{a} \subset X$ is a smooth irreducible divisor in $X$ which is linearly equivalent to $a H$ on $X$. As in Theorem [5.9, our aim is to show that if $\Sigma_{0} \subset S_{a}$ is a set of very general points on $S_{a}$, then we get a quadratic upper bound on $k$ for the admissible number $\delta_{0}=\left|\Sigma_{0}\right|$ in order for the complete linear system $\left|\mathcal{O}_{S_{a}}(k)\right|$ to separate $\Sigma_{0}$ on $S_{a}$.

Proposition 5.23. Let $X \subset \mathbb{P}^{r}$ be a smooth threefold of degree $d$ and let $H$ denote its hyperplane section. Assume that $\omega_{X} \cong \mathcal{O}_{X}(-m)$, for some integer $m \geq 0$. Let $S_{a}$ be a smooth irreducible divisor such that $S_{a} \sim a H$ on $X$, for some positive integer a. Denote by $H_{S_{a}}$ the hyperplane section of $S_{a} \subset \mathbb{P}^{r}$. Let $\Sigma_{0} \subset S_{a}$ be a set of $\delta_{0}$ distinct points on $S_{a}$. Given $k$ a non-negative integer, assume that:

(i) $k+m>a+2$, when either

a) $\delta_{0}=1$, or

b) $a d \geq 4$ and $2 \leq \delta_{0}<\frac{a d+\sqrt{a d(a d-4)}}{2}$;or

(ii) $k+m>a+\frac{4}{\sqrt{a d}}$, when either

a) $a d<4$ and $2 \leq \delta_{0}<\frac{(k+m-a)^{2}+\sqrt{(k+m-a)^{2} a d\left((k+m-a)^{2} a d-16\right)}}{8}$, or

b) $a d \geq 4$ and $\frac{a d+\sqrt{a d(a d-4)}}{2} \leq \delta_{0}<\frac{(k+m-a)^{2}+\sqrt{(k+m-a)^{2} a d\left((k+m-a)^{2} a d-16\right)}}{8}$.

Then,

$$
h^{1}\left(S_{a}, \mathcal{I}_{\Sigma_{0} / S_{a}}(k)\right)=0 .
$$

Proof. Let $b_{\delta_{0}}: \tilde{S}_{a} \rightarrow S_{a}$ be the blowing-up of $S_{a}$ along $\Sigma$. Then

$$
\begin{aligned}
H^{1}\left(S_{a}, \mathcal{I}_{\Sigma_{0} / S_{a}}(k)\right) & =H^{1}\left(S_{a}, \mathcal{I}_{\Sigma_{0} / S_{a}}(k+m-a) \otimes \omega_{S_{a}}\right) \\
& \cong H^{1}\left(\tilde{S}_{a}, \omega_{\tilde{S}_{a}} \otimes \mathcal{O}_{\tilde{S}_{a}}\left((k+m-a) b_{\delta_{0}}^{*}\left(H_{S_{a}}\right)-2 B\right)\right),
\end{aligned}
$$

where $B=\sum_{j=1}^{\delta_{0}} E_{j}$ is the $b_{\delta_{0}}$-exceptional divisor. Let $\tilde{\epsilon}=\epsilon\left(\mathcal{O}_{S_{a}}(1), \Sigma_{0}\right)$ be the multiple point Seshadri constant of $\mathcal{O}_{S_{a}}(1)$ at $\Sigma_{0}$; then

$$
(k+m-a) b_{\delta_{0}}^{*}\left(H_{S_{a}}\right)-2 B=\frac{2}{\tilde{\epsilon}}\left(b_{\delta_{0}}^{*}\left(H_{S_{a}}\right)-\tilde{\epsilon} B\right)+\left(k+m-a-\frac{2}{\tilde{\epsilon}}\right) b_{\delta_{0}}^{*}\left(H_{S_{a}}\right) .
$$

Therefore, if $\tilde{\epsilon}>\frac{2}{k+m-a}$ (with $a \neq k+m$ ), by the Kawamata-Viehweg vanishing theorem we get the desired vanishing in $(*)$.

If $\delta_{0}=1$, then $\epsilon\left(\mathcal{O}_{S_{a}}(1) ; p\right) \geq 1$ for each $p \in S_{a}$, since $\mathcal{O}_{S_{a}}(1)$ is very ample. Therefore, if $k+m>a+2$, the vanishing in (*) holds.

If $\delta_{0} \geq 2$, then, by Theorem 1.1 in [24], if $\epsilon\left(\mathcal{O}_{S_{a}}(1) ; \delta_{0}\right)$ denotes the multiple point Seshadri constant of $\mathcal{O}_{S_{a}}(1)$ at $\delta_{0}$ very general points of $S_{a}$, then

$$
\epsilon\left(\mathcal{O}_{S_{a}}(1) ; \delta_{0}\right) \geq M:=\min \left\{\epsilon\left(\mathcal{O}_{S_{a}}(1) ; 1\right), \frac{\sqrt{a d}}{2}, \frac{\sqrt{a d\left(\delta_{0}-1\right)}}{\delta_{0}}\right\} .
$$

By straightforward computations, if $\Sigma_{0} \subset S_{a}$ is a set of $\delta_{0}$ very general points on $S_{a}$ and if our numerical hypotheses hold, then the vanishing in $(*)$ holds.

As a consequence of Proposition 5.18 Remark 5.21 and Proposition 5.23 we have the following theorem. 
Theorem 5.25. Let $X \subset \mathbb{P}^{r}$ be a smooth threefold of degree $d$, and let $\mathcal{O}_{X}(1)$ denote its hyperplane bundle. Assume that $\omega_{X} \cong \mathcal{O}_{X}(-m)$, for some $m \geq 0$. Let $S_{a}$ be a smooth irreducible divisor on $X$ such that $S_{a} \in\left|\mathcal{O}_{X}(a)\right|$ on $X$ for some $a>0$. Let $[s] \in \mathcal{V}_{\delta}(\mathcal{E}(k))$, where $\mathcal{E}$ is a globally generated rank-two vector bundle on $X$ and $\delta$ and $k$ are positive integers. If $C=V(s)$ and $\Sigma=\operatorname{Sing}(C)$, assume that $\Sigma_{0} \subseteq \Sigma$ lies on $S_{a}$. Assume further that

$$
H^{1}\left(X, \mathcal{O}_{X}(k-a)\right)=H^{1}\left(X, \mathcal{O}_{X}(k)\right)=H^{2}\left(X, \mathcal{O}_{X}(k-a)\right)=(0)
$$

and that the numerical hypotheses in Proposition 5.23 hold. Then, if $[s] \in \mathcal{V}_{\delta}(\mathcal{E}(k))$ is not a regular point, the failure of the regularity property depends on the points in $\Sigma \backslash \Sigma_{0}$.

In particular, if $\Sigma=\Sigma_{0}$, then $[s] \in \mathcal{V}_{\delta}(\mathcal{E}(k))$ is a regular point.

Proof. From our assumptions, by Proposition 5.18 we get

$$
H^{1}\left(X, \mathcal{I}_{\Sigma_{0} / X}(k)\right) \cong H^{1}\left(X, \mathcal{I}_{\Sigma_{0} / S_{a}}(k)\right) .
$$

Now, from Proposition 5.23 and the exact sequence (5.22), it follows that

$$
H^{0}\left(\mathcal{O}_{\Sigma \backslash \Sigma_{0}}(k)\right) \rightarrow H^{1}\left(X, \mathcal{I}_{\Sigma / X}(k)\right),
$$

i.e., if there exists a non-zero obstruction, it is induced by an element in $H^{0}\left(\mathcal{O}_{\Sigma \backslash \Sigma_{0}}(k)\right)$.

When $X=\mathbb{P}^{3}$, the above results reduce to

Corollary 5.26. Let $\mathcal{E}$ be a globally generated rank-two vector bundle on $\mathbb{P}^{3}$ and let $k$ and $\delta$ be positive integers. Let $[s] \in \mathcal{V}_{\delta}(\mathcal{E}(k))$ and let $C=V(s)$ with $\Sigma=\operatorname{Sing}(C)$. Assume that $\Sigma_{0} \subseteq \Sigma$ lies on a smooth surface $S_{a}$ of degree $a$, and let $\delta_{0}$ be the cardinality of $\Sigma_{0}$. Assume that the points in $\Sigma_{0}$ are in very general position on $S_{a}$ and that one of the following conditions holds:

(i) $k>a-2$, when either

a) $a \geq 1$ and $\delta_{0}=1$, or

b) $a \geq 4$ and $2 \leq \delta_{0}<\frac{a+\sqrt{a(a-4)}}{2}$; or

(ii) $k>a-4+\frac{4}{\sqrt{a}}$, when either

a) $a<4$ and $2 \leq \delta_{0}<\frac{(k+4-a)^{2} a+\sqrt{(k+4-a)^{2} a\left((k+4-a)^{2} a-16\right)}}{8}$, or

b) $a \geq 4$ and $\frac{a+\sqrt{a(a-4)}}{2} \leq \delta_{0}<\frac{(k+4-a)^{2} a+\sqrt{(k+4-a)^{2} a\left((k+4-a)^{2} a-16\right)}}{8}$.

Then, $H^{1}\left(\mathbb{P}^{3}, \mathcal{I}_{\Sigma_{0} / \mathbb{P}^{3}}(k)\right)=(0)$. In particular, if $[s] \in \mathcal{V}_{\delta}(\mathcal{E}(k))$ fails to be a regular point, the failure of regularity depends on the nodes in $\Sigma \backslash \Sigma_{0}$. In particular, if $\Sigma=\Sigma_{0},[s] \in \mathcal{V}_{\delta}(\mathcal{E}(k))$ is a regular point.

Since we are interested in very general points, we can generalize the previous approach by assuming that $S_{a}=S$ is not necessarily smooth. Indeed, in general, if $\mu: \tilde{S} \rightarrow S$ denotes a resolution of singularities for $S$, given a Weil divisor $L$ on $S$, we have

$$
\epsilon\left(L ; \mu\left(p_{1}\right), \cdots, \mu\left(p_{\delta}\right)\right)=\epsilon\left(\mu^{*}(L) ; p_{1}, \ldots, p_{\delta}\right),
$$

since around the $p_{i}$ 's $\mu$ is an isomorphism. Therefore, we can more generally consider $S$ to be a normal surface with sufficiently mild singularities.

For simplicity, we shall discuss the case of $S \subset \mathbb{P}^{3}$ of degree $a$; the case $S \subset X \subset$ $\mathbb{P}^{r}$, where $X$ a smooth threefold, is a straightforward generalization. 
Theorem 5.28. Let $S \subset \mathbb{P}^{3}$ be an irreducible surface of degree a having at worst log-terminal singularities, and denote by $H$ the hyperplane section of $S$. Suppose that $K_{S}$ is a $\mathbb{Q}$-Cartier divisor of index $r$ such that $r K_{S} \equiv \alpha H$ for some $\alpha \in \mathbb{N}$. Let $\mathcal{E}$ be a globally generated rank-two vector bundle on $\mathbb{P}^{3}, k$ a positive integer and $[s] \in \mathcal{V}_{\delta}(\mathcal{E}(k))$. Let $C=V(s)$ and $\Sigma=\operatorname{Sing}(C)$. Assume that $\Sigma_{0} \subseteq \Sigma$ is a set of very general points in $S$. Assume further that

(i) $k>2+\frac{\alpha}{r}$, when either

a) $a \geq 1$ and $\delta_{0}=1$, or

b) $a>4$ and $2 \leq \delta_{0}<\frac{a+\sqrt{a(a-4)}}{2}$; or

(ii) $k>\frac{4}{\sqrt{a}}+\frac{\alpha}{r}$, when $a \leq 4$ and $\delta_{0}=2$,

(iii) $k>\frac{4 r}{\sqrt{a}}+\frac{\alpha}{r}$, when either

a) $a \leq 4$ and $3 \leq \delta_{0}<\frac{a(r k-\alpha)^{2}+\sqrt{a(r k-\alpha)^{2}\left(\left(a(r k-\alpha)^{2}-16 r^{2}\right)\right.}}{8 r^{2}}$, or

b) $a>4$ and $\frac{a+\sqrt{a(a-4)}}{2} \leq \delta_{0}<\frac{a(r k-\alpha)^{2}+\sqrt{a(r k-\alpha)^{2}\left(\left(a(r k-\alpha)^{2}-16 r^{2}\right)\right.}}{8 r^{2}}$.

Then, if $[s] \in \mathcal{V}_{\delta}(\mathcal{E}(k))$ is not a regular point, the failure of regularity depends on the nodes in $\Sigma \backslash \Sigma_{0}$. In particular, if $\Sigma=\Sigma_{0},[s] \in \mathcal{V}_{\delta}(\mathcal{E}(k))$ is a regular point.

Proof. Let $\mu: Y \rightarrow S$ be a log-resolution of the pair $(S, 0)$ (see $\S 1$ ). Then

$$
K_{Y}+\Delta \equiv \mu^{*}\left(K_{S}\right)+P
$$

where $\Delta$ is a boundary divisor and $P$ is an integral, effective and $\mu$-exceptional divisor on $Y$. Since $\Sigma_{0} \subset S$ is a set of very general points on $S$, then $\mu^{*}\left(\Sigma_{0}\right)=$ $\Sigma_{0}^{\prime} \cong \Sigma_{0}$ is a set of very general points on $Y$. Let $b_{\delta_{0}}: \tilde{Y} \rightarrow Y$ be the blowing-up of $Y$ along $\Sigma_{0}^{\prime}$, and denote by $F_{\delta_{0}}: \tilde{Y} \rightarrow S$ the composition $F_{\delta_{0}}=b_{\delta_{0}} \circ \mu$. Since, by hypothesis, $r K_{S} \equiv \alpha H$, then

$$
\begin{aligned}
h^{1}\left(S, \mathcal{I}_{\Sigma_{0} / S}(k)\right) & =h^{1}\left(S, \mathcal{I}_{\Sigma_{0} / S}\left(\left(k-\frac{\alpha}{r}\right) H+K_{S}\right)\right) \\
& =h^{1}\left(Y, \mathcal{I}_{\Sigma_{0}^{\prime} / Y} \otimes \mathcal{O}_{Y}\left(\left(\left(k-\frac{\alpha}{r}\right) \mu^{*}(H)+K_{Y}+\Delta\right)\right)\right) \\
& =h^{1}\left(\tilde{Y}, \mathcal{O}_{\tilde{Y}}\left(K_{\tilde{Y}}+b_{\delta_{0}}^{*}(\Delta)+\left(k-\frac{\alpha}{r}\right) F_{\delta_{0}}^{*}(H)-2 B\right)\right),
\end{aligned}
$$

where $B=\sum_{i=1}^{\delta_{0}} E_{i}$ is the $b_{\delta_{0}}$-exceptional divisor. Since, by (5.27),

$$
\bar{\epsilon}=\epsilon\left(\mu^{*}\left(\mathcal{O}_{S}(1)\right), \Sigma_{0}^{\prime}\right)=\epsilon\left(\mathcal{O}_{S}(1), \Sigma_{0}\right),
$$

then

$$
\left(k-\frac{\alpha}{r}\right) F_{\delta_{0}}^{*}(H)-2 B=\left(k-\frac{\alpha}{r}-\frac{2}{\bar{\epsilon}}\right) F_{\delta_{0}}^{*}(H)+\frac{2}{\bar{\epsilon}}\left(F_{\delta_{0}}^{*}(H)-\bar{\epsilon} B\right)
$$

is big and nef if $k-\frac{\alpha}{r}>\frac{2}{\bar{\epsilon}}$, i.e. if $\bar{\epsilon}>\frac{2 r}{r k-\alpha}$. At this point, we can apply the same computations as in Proposition 5.23 and Theorem 5.25 .

Remark 5.29. As observed in Remark 5.16 for sets of points in very general position on a threefold, from Corollary 5.26 and Theorem 5.28 we see that, when $\Sigma_{0}=\Sigma$ is assumed to be a set of very general points lying on a smooth surface in $\mathbb{P}^{3}$ or on a normal surface with at worst log-terminal singularities, there are upper bounds on the number of admissible points in $\Sigma$ such that an element $[s] \in \mathcal{V}_{\delta}(\mathcal{E}(k))$ whose zero-locus $C$ has nodes at $\Sigma$ is a regular point. Such upper bounds are quadratic polynomials in $k$; this reflects the fact that $\Sigma$ is assumed to be a set of very general points lying on a 2-dimensional subscheme. Indeed, all the computations are related to multiple point Seshadri constants of very ample line bundles on such schemes. 
Looking back at Proposition 5.23 at Theorem 5.28 and at Corollary 5.26] we want to study how the upper bounds on $\delta_{0}=\left|\Sigma_{0}\right|$ vary when we drop the hypothesis that $\Sigma_{0}$ is a set of very general points on $S$. To get some effective results, we can apply the techniques in [3] for smooth surfaces, and their generalizations in [23] for normal surfaces. These techniques are both generalizations of Reider's theorem (see [31]).

We first recall some standard and useful definitions (see (0.1) in [3]).

Definition 5.30. Let $S$ be a smooth projective surface. A line bundle $L$ on $S$ is said to be $k$-very ample, $k \geq 0$, if the restriction map

$$
H^{0}(S, L) \rightarrow H^{0}\left(Z, \mathcal{O}_{Z}(L)\right)
$$

is surjective for any $Z \in \mathcal{H}_{i l b} b^{k+1}(S)$.

As an immediate consequence of the definition, we have the following generalization of Proposition 4.4 to the surface case.

Lemma 5.31. If $L_{1}, \cdots, L_{k}$ are very ample line bundles on $S, L_{1} \otimes \cdots \otimes L_{k}$ is k-very ample.

Proof. See Lemma 0.1.1 in [3].

Corollary 5.32. Let $X \subset \mathbb{P}^{r}$ be a smooth threefold and let $\mathcal{O}_{X}(1)$ denote its hyperplane bundle. Let $\mathcal{E}$ be a globally generated rank-two vector bundle on $X$ and let $[s] \in \mathcal{V}_{\delta}(\mathcal{E}(k))$, where $k$ and $\delta$ are positive integers. Let $C=V(s), \Sigma=\operatorname{Sing}(C)$, and assume that $\Sigma_{0} \subseteq \Sigma$ lies on a smooth divisor $S \subset X$ whose hyperplane section is denoted by $H_{S}$. Assume further that

$$
h^{1}\left(X, \mathcal{O}_{X}(k)\right)=h^{1}\left(X, \mathcal{I}_{S / X}(k)\right)=h^{2}\left(X, \mathcal{I}_{S / X}(k)\right)=0 .
$$

Then, if $\delta_{0} \leq k+1$, we have $h^{1}\left(X, \mathcal{I}_{\Sigma_{0} / X}(k)\right)=0$. Therefore, if $[s] \in \mathcal{V}_{\delta}(\mathcal{E}(k))$ fails to be a regular point, the failure of regularity depends on the nodes in $\Sigma \backslash \Sigma_{0}$. In particular, if $\Sigma=\Sigma_{0}$, then $[s] \in \mathcal{V}_{\delta}(\mathcal{E}(k))$ is regular.

Proof. Since $\mathcal{O}_{S}\left(k H_{S}\right)=\mathcal{O}_{S}\left(H_{S}\right)^{\otimes k}$ and since $\mathcal{O}_{S}\left(H_{S}\right)$ is very ample on $S$, then $\mathcal{O}_{S}\left(k H_{S}\right)$ is $\left(\delta_{0}-1\right)$-very ample if $k \geq \delta_{0}-1$. One concludes by using Proposition 5.18 and Remark 5.21.

Remark 5.33. Observe that, since there is no assumption on the postulation of points in $\Sigma_{0}$, we re-find a uniform and linear upper bound on $\delta_{0}$ as we determined in Proposition 4.4 and in Theorem 4.5 for $\Sigma \subset X$, where $X$ a smooth, projective threefold.

Proposition 5.34. Let $X \subset \mathbb{P}^{r}$ be a smooth threefold of degree $d$ and let $\mathcal{O}_{X}(1)$ denote its hyperplane bundle. Assume that $\omega_{X} \cong \mathcal{O}_{X}(-m)$ for some non-negative integer $m$. Let $\mathcal{E}$ be a globally generated rank-two vector bundle on $X$, let $k$ and $\delta$ be positive integers, and let $[s] \in \mathcal{V}_{\delta}(\mathcal{E}(k))$. Let $C=V(s), \Sigma=\operatorname{Sing}(C)$, and assume that $\Sigma_{0} \subseteq \Sigma$ lies on a smooth irreducible divisor $S \sim a H$ on $X$. Denote by $H_{S}$ the hyperplane section of $S$. Assume further that

$$
h^{1}\left(X, \mathcal{O}_{X}(k-a)\right)=h^{1}\left(X, \mathcal{O}_{X}(k)\right)=h^{2}\left(X, \mathcal{O}_{X}(k-a)\right)=0 .
$$

If $k+m>a$ and $\delta_{0} \leq \frac{k+m-a}{2} \operatorname{deg}(D)$, for each curve $D$ on $S$, then

$$
h^{1}\left(X, \mathcal{I}_{\Sigma_{0} / X}(k)\right)=0
$$


If, in particular, $N S(S) \cong \mathbb{Z}\left[H_{S}\right]$, then

$$
h^{1}\left(X, \mathcal{I}_{\Sigma_{0} / X}(k)\right)=0 \quad \text { when } \delta_{0} \leq \frac{\operatorname{ad}(k+m-a)}{2} .
$$

Therefore, if $[s] \in \mathcal{V}_{\delta}(\mathcal{E}(k))$ fails to be a regular point, the failure of regularity depends on the behaviour of the nodes in $\Sigma \backslash \Sigma_{0}$. In particular, if $\Sigma=\Sigma_{0}$, then $[s] \in \mathcal{V}_{\delta}(\mathcal{E}(k))$ is a regular point.

Proof. Consider $L=\mathcal{O}_{S}\left(k H_{S}-K_{S}\right)=\mathcal{O}_{S}(k+m-a)$. Since $k+m>a, L$ is very ample on $S$ and $L D=(k+m-a) \operatorname{deg}(D)$, for each curve $D$ on $S$. By Theorem 2.1 and Corollary 2.3 in [3], if $\delta_{0} \leq \frac{k+m-a}{2} \operatorname{deg}(D)$, then

$$
H^{0}\left(S, \mathcal{O}_{S}(k)\right)=H^{0}\left(S, \mathcal{O}_{S}\left(K_{S}+L\right)\right) \rightarrow H^{0}\left(\mathcal{O}_{\Sigma_{0}}\right),
$$

for $\Sigma_{0} \in \mathcal{H} i l b^{\delta_{0}}(S)$. Since $L$ is very ample on $S$, by the Kodaira vanishing and by the surjectivity above, we have that $h^{1}\left(S, \mathcal{I}_{\Sigma_{0} / S}(k)\right)=0$. From our assumption (*), it follows that $h^{1}\left(X, \mathcal{I}_{\Sigma_{0} / X}(k)\right)=0$.

Remark 5.35. Observe that Proposition 5.34 applies, in particular, to general surfaces in $\mathbb{P}^{3}$ of degree $a \geq 4$.

By adapting the procedure of Theorem 3 in 23 to our situation, we can easily extends to the case when $S$ is a normal surface and prove analogous results. For brevity, the interested reader is referred to 23 .

We conclude this section by studying the case when, given $[s] \in \mathcal{V}_{\delta}(\mathcal{E}(k))$ and $C=V(s)$, some nodes of $C$ are assumed to be on a given curve $\Gamma \subset X$. As expected, we find linear bounds in $k$ for the number of admissible nodes of $C$ lying on $\Gamma$ in order for $\mathcal{O}_{X}(k)$ to separate such points on $\Gamma$. Precisely, we have:

Theorem 5.36. Let $X \subset \mathbb{P}^{r}$ be a smooth threefold and let $\mathcal{O}_{X}(1)$ denote its hyperplane bundle. Let $\mathcal{E}$ be a globally generated rank-two vector bundle on $X$, and let $k$ and $\delta$ be positive integers. Consider $[s] \in \mathcal{V}_{\delta}(\mathcal{E}(k))$ and let $C=V(s)$. Take $\Sigma_{0} \subseteq \Sigma=\operatorname{Sing}(C),\left|\Sigma_{0}\right|=\delta_{0}$, a (not necessarily proper) subset of its nodes. Assume that $\Sigma_{0}$ lies on a local complete intersection curve $\Gamma \subset X$ such that its dualizing sheaf is $\omega_{\Gamma} \cong \mathcal{O}_{\Gamma}(e)$, for some $e \in \mathbb{Z}$. Assume that $h^{1}\left(X, \mathcal{O}_{X}(k)\right)=0$ and that:

(i) $k>e$,

(ii) $h^{1}\left(X, \mathcal{I}_{\Gamma / X}(k)\right)=0$, and

(iii) $\delta_{0}<\operatorname{deg}\left(\mathcal{O}_{\Gamma}(k-e)\right)$.

Then $h^{1}\left(X, \mathcal{I}_{\Sigma_{0} / X}(k)\right)=0$. Therefore, if $[s] \in \mathcal{V}_{\delta}(\mathcal{E}(k))$ fails to be a regular point, the failure depends on the behaviour of the nodes in $\Sigma \backslash \Sigma_{0}$. In particular, if $\Sigma=\Sigma_{0}$, then $[s] \in \mathcal{V}_{\delta}(\mathcal{E}(k))$ is a regular point.

Proof. Consider the ideal sequence

$$
0 \rightarrow \mathcal{I}_{\Gamma / X}(k) \rightarrow \mathcal{I}_{\Sigma_{0} / X}(k) \rightarrow \mathcal{I}_{\Sigma_{0} / \Gamma}(k) \rightarrow 0 .
$$

Since $h^{1}\left(X, \mathcal{I}_{\Gamma / X}(k)\right)=0$, then

$$
h^{1}\left(\mathcal{I}_{\Sigma_{0} / X}(k)\right) \hookrightarrow h^{1}\left(\mathcal{I}_{\Sigma_{0} / \Gamma}(k)\right) .
$$

Therefore a sufficient condition will be the vanishing $h^{1}\left(\mathcal{I}_{\Sigma_{0} / \Gamma}(k)\right)=0$. As in [16], consider that $\left.H^{1}\left(\mathcal{I}_{\Sigma_{0} / \Gamma}(k)\right)^{\vee} \cong \operatorname{Hom}\left(\mathcal{I}_{\Sigma_{0} / \Gamma}(k)\right), \omega_{\Gamma}\right)$. 
Assume, for a contradiction, that $h^{1}\left(X, \mathcal{I}_{\Sigma_{0} / X}(k)\right) \neq 0$; then a non-zero element corresponds to a non-zero sheaf morphism

$$
\mathcal{I}_{\Sigma_{0} / \Gamma}(k) \stackrel{\varphi}{\rightarrow} \omega_{\Gamma}
$$

Since $\mathcal{I}_{\Sigma_{0} / \Gamma}(k) \subset \mathcal{O}_{\Gamma}(k)$ is a torsion-free (but not locally free) sheaf on $\Gamma$ of rank one, $\varphi$ is an injective sheaf morphism. Thus,

$$
0 \rightarrow \mathcal{I}_{\Sigma_{0} / \Gamma}(k) \rightarrow \omega_{\Gamma} \rightarrow \underline{t} \rightarrow 0,
$$

where $\underline{t}$ is a torsion sheaf on $\Gamma$. Therefore, if $\chi(-)$ denotes the Euler characteristic,

$$
0 \leq \chi(\underline{t})=\chi\left(\omega_{\Gamma}\right)-\chi\left(\mathcal{I}_{\Sigma_{0} / \Gamma}(k)\right)=p_{a}(\Gamma)-1-\chi\left(\mathcal{O}_{\Gamma}(k)\right)+\delta_{0},
$$

i.e. $\delta_{0} \geq \chi\left(\mathcal{O}_{\Gamma}(k)\right)-p_{a}(\Gamma)+1=\operatorname{deg}\left(\mathcal{O}_{\Gamma}(k-e)\right)>0$, since $k>e$ by assumption. Therefore, if $h^{1}\left(\mathcal{I}_{\Sigma_{0} / \Gamma}(k)\right) \neq 0, \delta_{0}$ must be greater than or equal to $\operatorname{deg}\left(\mathcal{O}_{\Gamma}(k-e)\right)$, which contradicts our hypotheses.

Corollary 5.37. Let $\mathcal{E}$ be a globally generated rank-two vector bundle on $\mathbb{P}^{3}$, and let $k$ and $\delta$ be positive integers. Consider $[s] \in \mathcal{V}_{\delta}(\mathcal{E}(k))$, and let $C=V(s)$. Take $\Sigma_{0} \subseteq \Sigma=\operatorname{Sing}(C)$, a subset of the nodes of $C$ s.t $\left|\Sigma_{0}\right|=\delta_{0}$. Assume that $\Sigma_{0}$ lies on a complete intersection curve $\Gamma_{b} \subset \mathbb{P}^{3}$ of degree $b$ such that $\omega_{\Gamma_{b}} \cong \mathcal{O}_{\Gamma_{b}}(e)$, for some $e \in \mathbb{Z}$. Assume that $k>e$ and $\delta_{0}<b(k-e)$. Then $h^{1}\left(X, \mathcal{I}_{\Sigma_{0} / \mathbb{P}^{3}}(k)\right)=0$.

Therefore, if $[s] \in \mathcal{V}_{\delta}(\mathcal{E}(k))$ fails to be a regular point, the failure depends on the behaviour of the nodes in $\Sigma \backslash \Sigma_{0}$. In particular, if $\Sigma=\Sigma_{0}$, then $[s] \in \mathcal{V}_{\delta}(\mathcal{E}(k))$ is a regular point.

Proof. Since $\Gamma_{b}$ is a complete intersection, then $h^{1}\left(\mathcal{I}_{\Gamma / \mathbb{P}^{3}}(k)\right)=0$, for each $k$. Moreover, $\operatorname{deg}\left(\mathcal{O}_{\Gamma_{b}}(k-e)\right)=b(k-e)$.

Remark 5.38. Observe that, with Theorem 5.36] and Corollary 5.37, one can very easily construct several examples of curves on a threefold $X$ or, more specifically, in $\mathbb{P}^{3}$ which correspond to non-regular points of $\mathcal{V}_{\delta}(\mathcal{E}(k))$, proving the almost sharpness of our bounds. For example, Corollary 5.37 gives a complete generalization of Example 3.2 in [1. The authors of [1] considered $\mathcal{E}=\mathcal{O}_{\mathbb{P}^{3}}(1) \oplus \mathcal{O}_{\mathbb{P}^{3}}(4)$ and $\delta=$ $k+4$, with $k>>0$; they constructed a curve $C \subset \mathbb{P}^{3}$, corresponding to a point $[s] \in \mathcal{V}_{k+4}(\mathcal{E}(k))$, with $\Sigma_{0}=\Sigma=\operatorname{Sing}(C)$ is a set of $k+4$ nodes lying on a line $L$, and they showed that $[s]$ is not a regular point of $\mathcal{V}_{\delta}(\mathcal{E}(k))$. Such an example is a particular case of our result, since for the regularity of $[s]$, we showed that one must require that the number of nodes lying on $L$ must be $\delta_{0}<1(k-(-2))=k+2$. Observe also that our Corollary 5.37 holds not only for $k>>0$ but for effective values of $k$, and, furthermore, that we can replace the line $L$ by any other complete intesection curve in $\mathbb{P}^{3}$.

\section{SOME GEOMETRIC PROPERTIES OF SPACE CURVES PARAMETRIZED BY $\mathcal{V}_{\delta}(\mathcal{E}(k))$}

From now on, we shall consider only curves in $\mathbb{P}^{3}$. Let $\left[s_{0}\right] \in \mathcal{V}_{\delta}(\mathcal{E}(k))$, where $\mathcal{E}$ is a globally generated rank-two vector bundle on $\mathbb{P}^{3}$ and where $k$ and $\delta$ are positive integers. Let $C=V\left(s_{0}\right) \subset \mathbb{P}^{3}$, and let $\Sigma$ denote the set of nodes of $C$.

The aim of this section is to study some interesting geometric properties of the curves determined by elements in $\mathcal{V}_{\delta}(\mathcal{E}(k))$. Precisely, given an integer $h>0$, we can consider smooth curves $\Gamma_{h} \subset \mathbb{P}^{3}$, which are defined as zero-loci of suitable global sections $s_{h} \in H^{0}\left(\mathbb{P}^{3}, \mathcal{E}(k+h)\right)$; then we want to study geometric properties 
of the pairs $\left(C, \Gamma_{h}\right), h \in \mathbb{N}$, expecially from the liaison relation point of view. From Rao's paper (30]), we know that $C$ and $\Gamma_{h}$ lie in the same liaison class, for each $h>0$. Indeed, since such curves correspond to global sections of twists of the same vector bundle $\mathcal{E}$, by the Koszul exact sequences in $\mathbb{P}^{3}$, their Rao modules are isomorphic up to the shift of $h$, i.e.

$$
M(C)=\bigoplus_{t} H^{1}\left(C, \mathcal{I}_{C / \mathbb{P}^{3}}(t)\right) \cong \bigoplus_{t} H^{1}\left(\Gamma_{h}, \mathcal{I}_{C / \mathbb{P}^{3}}(h+t)\right)=M\left(\Gamma_{h}\right) .
$$

One can be more precise by recalling the following terminology.

Definition 6.2 (see Def. 1.1 in [30]). Let $V_{1}, V_{2} \subset \mathbb{P}^{3}$ be two subschemes which are locally Cohen-Macaulay and equidimensional of codimensione two. Denote by $\sim_{l}$ the relation of (geometric) linkage in $\mathbb{P}^{3}$. Then $V_{1}$ and $V_{2}$ are in the same even liaison class if

$$
V_{1} \sim_{l} Z_{1} \sim_{l} \cdots \sim_{l} Z_{2 k+1} \sim_{l} V_{2}
$$

for some schemes $Z_{i} \subset \mathbb{P}^{3}$. In such a case, $V_{1}$ and $V_{2}$ are said to be evenly linked. The resulting equivalence relation is called biliaison.

By (6.1) and by Lemma 1.6 in [30, we immediately observe that the curves $C$ and $\Gamma_{h}$ lie in the same biliaison class, apart from some particular cases. Indeed, since $C$ and $\Gamma_{h}$ are both subcanonical curves in $\mathbb{P}^{3}$, it may happen that $C$ and $\Gamma_{h}$ are directly (so oddly) linked in $\mathbb{P}^{3}$ (see [5]). However, when this last situation occurs, there are strong restrictions not only on the vector bundle $\mathcal{E}$ but also on the numerical characters of $\Gamma_{h}$ (degree, genus and postulation); precisely, one has at most three possible cases for $\Gamma_{h}$ (for details, the reader is referred to the original paper [5]).

Here we are interested in analyzing the geometric properties that $C$ and $\Gamma_{h}$ share, for each $h>0$, particularly towards the biliaison relation between $C$ and $\Gamma_{h}$ (i.e. with general choices of $\mathcal{E}$ and $\Gamma_{h}$ ).

By recalling Remark 2.3, we get the following first result.

Theorem 6.3. Let $\mathcal{E}$ be a globally generated rank-two vector bundle on $\mathbb{P}^{3}$, and denote by $c_{i}$ the $i^{\text {th }}$ Chern class of $\mathcal{E}, 1 \leq i \leq 2$. Let $k$ and $\delta$ be positive integers such that $\delta \leq k+1$. Let $\left[s_{0}\right] \in \mathcal{V}_{\delta}(\mathcal{E}(k))$ correspond to an irreducible nodal curve $C \subset \mathbb{P}^{3}$ whose set of nodes is denoted by $\Sigma$. Take $h \geq 1$ an integer such that

$$
k+h \geq c_{1} \in \mathbb{Z} \text {. }
$$

Then, there always exist:

(i) a smooth, irreducible curve $\Gamma_{h} \subset \mathbb{P}^{3}$ simply passing through $\Sigma$ and such that $\Gamma_{h}=V\left(s_{h}\right)$, where $\left[s_{h}\right] \in \mathbb{P}\left(H^{0}(\mathcal{E}(k+h))\right.$, and

(ii) a normal surface $S_{k, h} \subset \mathbb{P}^{3}$ of degree $d_{k, h}=c_{1}+2 k+h$ containing both $C$ and $\Gamma_{h}$.

Proof. As in Theorem 3.4 we consider the smooth projective fourfold $\mathcal{P}=\mathbb{P}_{\mathbb{P}^{3}}(\mathcal{E}(k))$, together with the surjective morphism $\pi: \mathcal{P} \rightarrow \mathbb{P}^{3}$ and the tautological line bundle $\mathcal{O}_{\mathcal{P}}(1)$. Thus the curve $C$, which is the zero-locus of the global section $s_{0} \in H^{0}(\mathcal{E}(k))$, corresponds to an effective divisor $D_{0} \in\left|\mathcal{O}_{\mathcal{P}}(1)\right|$.

(i) Given $h>0$, we first want to construct a section $s_{h} \in H^{0}(\mathcal{E}(k+h))$ which corresponds to a divisor $D_{h} \in\left|\mathcal{O}_{\mathcal{P}}(1) \otimes \pi^{*}\left(\mathcal{O}_{\mathbb{P}^{3}}(h)\right)\right|$ simply vanishing along the lines of the scheme $\mathcal{L}=\bigcup_{i=1}^{\delta} L_{i}:=\pi^{-1}(\Sigma)$. Such a divisor will correspond to the curve $\Gamma_{h}$ we want to determine. 
Recall that the divisor $D_{0}$ corresponding to $C$ is singular, having $\delta$ rational double points at $\Sigma^{1} \subset \mathcal{L}$, where $\Sigma^{1} \cong \Sigma$ (see the proof of Theorem 3.4). Assume for a moment that the sheaf

$$
\mathcal{I}_{\mathcal{L} / \mathcal{P}} \otimes \mathcal{O}_{\mathcal{P}}(1) \otimes \pi^{*}\left(\mathcal{O}_{\mathbb{P}^{3}}(h)\right)
$$

is globally generated on $\mathcal{P}$ (we shall show this fact later on in this proof); thus, the scheme $\mathcal{L}$ coincides with the base locus of the linear system

$$
\left|\mathcal{I}_{\mathcal{L} / \mathcal{P}} \otimes \mathcal{O}_{\mathcal{P}}(1) \otimes \pi^{*}\left(\mathcal{O}_{\mathbb{P}^{3}}(h)\right)\right|
$$

Since $H^{0}(\mathcal{E}(k)) \hookrightarrow H^{0}(\mathcal{E}(k+h))$, for a general $\sigma \in H^{0}\left(\mathcal{O}_{\mathbb{P}^{3}}(h)\right)$ the global section $s_{0} \otimes \sigma \in H^{0}(\mathcal{E}(k+h))$ behaves as $s_{0}$ around the points in $\Sigma$; moreover, since

$$
\pi_{*}\left(\mathcal{O}_{\mathcal{P}}(1) \otimes \pi^{*}\left(\mathcal{O}_{\mathbb{P}^{3}}(h)\right)\right) \cong \mathcal{E}(k+h),
$$

if

$$
B:=\text { Base scheme }\left(\left|\mathcal{I}_{\mathcal{L} / \mathcal{P}} \otimes \mathcal{O}_{\mathcal{P}}(1) \otimes \pi^{*}\left(\mathcal{O}_{\mathbb{P}^{3}}(h)\right)\right|\right)
$$

then $\mathcal{L}=B$ as schemes, which means that each line $L_{i}$ is reduced in $B$.

We want to show that the general element of $\left|\mathcal{I}_{\mathcal{L} / \mathcal{P}} \otimes \mathcal{O}_{\mathcal{P}}(1) \otimes \pi^{*}\left(\mathcal{O}_{\mathbb{P}^{3}}(h)\right)\right|$ is smooth along $\mathcal{L}$. Denote by $D_{h}$ the general divisor of $\left|\mathcal{I}_{\mathcal{L} / \mathcal{P}} \otimes \mathcal{O}_{\mathcal{P}}(1) \otimes \pi^{*}\left(\mathcal{O}_{\mathbb{P}^{3}}(h)\right)\right|$; since we assumed that the sheaf in (6.5) is globally generated, then

$$
\mathcal{N}_{\mathcal{L} / D_{h}}^{\vee} \otimes \mathcal{O}_{\mathcal{P}}\left(D_{h}\right) \cong \frac{\mathcal{I}_{\mathcal{L} / \mathcal{P}}}{\mathcal{I}_{\mathcal{L} / \mathcal{P}}^{2}} \otimes \mathcal{O}_{\mathcal{P}}(1) \otimes \pi^{*}\left(\mathcal{O}_{\mathbb{P}^{3}}(h)\right)
$$

is globally generated. Since we have $\mathcal{L} \subset D_{h} \subset \mathcal{P}$, we get

$(6.6) \quad 0 \rightarrow \mathcal{O}_{D_{h}} \cong \mathcal{N}_{D_{h} / \mathcal{P}}^{\vee} \otimes \mathcal{O}_{\mathcal{P}}\left(D_{h}\right) \rightarrow \mathcal{N}_{\mathcal{L} / \mathcal{P}}^{\vee} \otimes \mathcal{O}_{\mathcal{P}}\left(D_{h}\right) \rightarrow \mathcal{N}_{\mathcal{L} / D_{h}}^{\vee} \otimes \mathcal{O}_{\mathcal{P}}\left(D_{h}\right) \rightarrow 0$.

Fix $L=L_{i_{0}} \subset \mathcal{L}$ for some $1 \leq i_{0} \leq \delta$, and restrict (6.6) to $L$; therefore we have

$$
\mathcal{O}_{L} \rightarrow \mathcal{N}_{\mathcal{L} / \mathcal{P}}^{\vee} \otimes \mathcal{O}_{L}\left(D_{h}\right) \rightarrow \mathcal{N}_{\mathcal{L} / D_{h}}^{\vee} \otimes \mathcal{O}_{L}\left(D_{h}\right) \rightarrow 0
$$

Since $\mathcal{N}_{\mathcal{L} / \mathcal{P}}^{\vee} \otimes \mathcal{O}_{L}\left(D_{h}\right)$ is a globally generated rank-three vector bundle on the line $L$, there exists a global section nowhere vanishing on $L$; this implies that (6.7) is exact and that $\mathcal{N}_{\mathcal{L} / \mathcal{P}}^{\vee} \otimes \mathcal{O}_{L}\left(D_{h}\right)$ is locally free on $L$. So it is $\left.\mathcal{N}_{\mathcal{L} / \mathcal{P}}^{\vee}\right|_{L}$. Since we have $L \subset \mathcal{L} \subset D_{h}$, then also $\mathcal{N}_{L / D_{h}}^{\vee}$ is locally free. Therefore, since $L$ is smooth in $\mathcal{P}$, we have

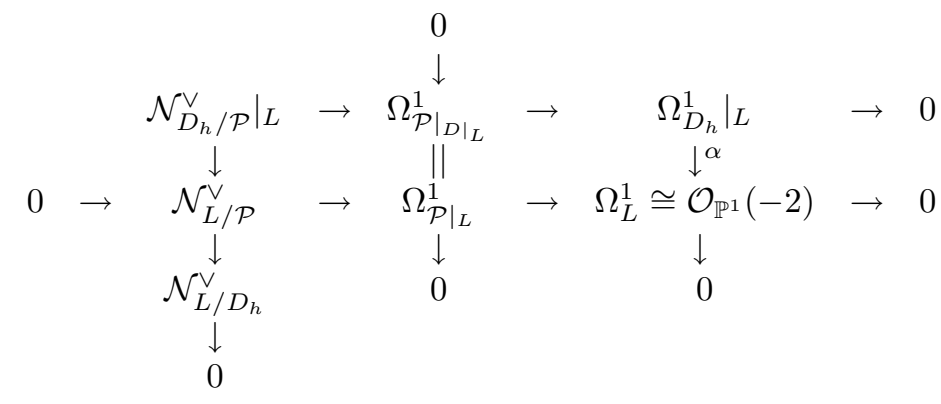

By the snake lemma, $\operatorname{ker}(\alpha) \cong \mathcal{N}_{L / D_{h}}^{\vee}$ is locally free on $L$. This implies that $\left.\Omega_{D_{h}}^{1}\right|_{L}$ is locally free on $L$, i.e. the general element of $\left|\mathcal{I}_{\mathcal{L} / \mathcal{P}} \otimes \mathcal{O}_{\mathcal{P}}(1) \otimes \pi^{*}\left(\mathcal{O}_{\mathbb{P}^{3}}(h)\right)\right|$ is smooth on $L$.

To complete the proof, we only have to show that the sheaf in (6.5) is actually globally generated on $\mathcal{P}$. Observe that $\mathcal{I}_{\mathcal{L} / \mathcal{P}} \otimes \mathcal{O}_{\mathcal{P}}(1) \otimes \pi^{*}\left(\mathcal{O}_{\mathbb{P}^{3}}(h)\right)$ is globally generated iff $\mathcal{I}_{\Sigma / \mathbb{P}^{3}} \otimes \mathcal{E}(k+h)$ is globally generated on $\mathbb{P}^{3}$. Since a sufficient condition 
for the global generation of $\mathcal{I}_{\Sigma / \mathbb{P}^{3}} \otimes \mathcal{E}(k+h)$ is its 0-regularity as a sheaf on $\mathbb{P}^{3}$, we observe that $\mathcal{I}_{\Sigma / \mathbb{P}^{3}} \otimes \mathcal{E}(k+h)$ is 0 -regular iff (6.8)

$h^{1}\left(\mathcal{I}_{\Sigma / \mathbb{P}^{3}} \otimes \mathcal{E}(k+h-1)\right)=h^{2}\left(\mathcal{I}_{\Sigma / \mathbb{P}^{3}} \otimes \mathcal{E}(k+h-2)\right)=h^{3}\left(\mathcal{I}_{\Sigma / \mathbb{P}^{3}} \otimes \mathcal{E}(k+h-3)\right)=0$.

From the Griffiths vanishing result (see [34], page 107), it follows that

$$
h^{1}(\mathcal{E}(k+h-1))=h^{2}(\mathcal{E}(k+h-2))=h^{3}(\mathcal{E}(k+h-3))=0,
$$

since $\mathcal{E}$ is globally generated and since $k+h \geq c_{1}=\operatorname{det}(\mathcal{E})$ by assumption. Therefore, from the exact sequence

$$
\left.0 \rightarrow \mathcal{I}_{\Sigma / \mathbb{P}^{3}} \otimes \mathcal{E}(k+h) \rightarrow \mathcal{E}(k+h) \rightarrow \mathcal{E}(k+h)\right|_{\Sigma} \rightarrow 0
$$

and from (6.9), the last two equalities in (6.8) hold. It only remains to show that $h^{1}\left(\mathcal{I}_{\Sigma / \mathbb{P}^{3}} \otimes \mathcal{E}(k+h-1)\right)=0$. To this aim, take

$$
\begin{aligned}
0 & \rightarrow H^{0}\left(\mathcal{I}_{\Sigma / \mathbb{P}^{3}} \otimes \mathcal{E}(k+h-1)\right) \rightarrow H^{0}(\mathcal{E}(k+h-1)) \\
& \stackrel{\alpha_{k+h-1}}{\longrightarrow} H^{0}\left(\left.\mathcal{E}(k+h-1)\right|_{\Sigma}\right) \rightarrow H^{1}\left(\mathcal{I}_{\Sigma / \mathbb{P}^{3}} \otimes \mathcal{E}(k+h-1)\right) \rightarrow 0 .
\end{aligned}
$$

Since $\delta \leq k+1$,

$$
H^{0}(\mathcal{E}(k)) \stackrel{\alpha_{k}}{\longrightarrow} H^{0}\left(\left.\mathcal{E}(k)\right|_{\Sigma}\right)
$$

is surjective; therefore, $\alpha_{k+h-1}$ is surjective since $h \geq 1$, i.e.

$$
H^{1}\left(\mathcal{I}_{\Sigma / \mathbb{P}^{3}} \otimes \mathcal{E}(k+h-1)\right)=(0)
$$

(ii) After having constructed the curve $\Gamma_{h}$, which corresponds to the general element of $\left|\mathcal{I}_{\mathcal{L} / \mathcal{P}} \otimes \mathcal{O}_{\mathcal{P}}(1) \otimes \pi^{*}\left(\mathcal{O}_{\mathbb{P}^{3}}(h)\right)\right|$, take $s_{h} \in H^{0}(\mathcal{E}(k+h))$ such that $\Gamma_{h}=$ $V\left(s_{h}\right)$. We can consider the rank-two vector bundle morphism

$$
\tau=\left(s_{0}, s_{h}\right): \mathcal{O}_{\mathbb{P}^{3}} \oplus \mathcal{O}_{\mathbb{P}^{3}}(-h) \rightarrow \mathcal{E}(k),
$$

where $s_{0} \in H^{0}(\mathcal{E}(k))$ is such that $C=V\left(s_{0}\right)$. The degeneration locus of the morphism $\tau$ is a surface $S_{k, h}=V(\operatorname{det}(\tau))$, where

$$
\operatorname{det}(\tau) \in H^{0}\left(\mathbb{P}^{3}, \operatorname{det}(\mathcal{E}) \otimes \mathcal{O}_{\mathbb{P}^{3}}(2 k+h)\right) .
$$

Therefore, $S_{k, h}$ is a surface of degree $d_{k, h}=c_{1}+2 k+h$ containing both $C=V\left(s_{0}\right)$ and $\Gamma_{h}=V\left(s_{h}\right)$. Its singular locus is determined by the condition $\operatorname{rank}(\tau)<$ 1; therefore, by the construction of $\Gamma_{h}$ in (i), we immediately observe that $\Sigma \subseteq$ $\operatorname{Supp}\left(C \cap \Gamma_{h}\right)=\operatorname{Sing}\left(S_{k, h}\right)$.

For each $k$ and $h$ as in (6.4), the surface $S_{k, h}$ constructed in Theorem 6.3 is the most "natural" surface in $\mathbb{P}^{3}$ containing both curves $C$ and $\Gamma_{h}$. One can very easily deduce some biliaison properties of $C$ and $\Gamma_{h}$ on $S_{k, h}$. We first recall the following more general definition from [21.

Definition 6.10. Let $V_{1}, V_{2}$ be schemes of equidimension one without embedded components which are evenly linked in $\mathbb{P}^{3} . V_{2}$ is said to be obtained from $V_{1}$ by an elementary biliaison of height $h$, for some $h \in \mathbb{Z}$, if there exists a surface $S \subset \mathbb{P}^{3}$ containing $V_{1}$ and $V_{2}$ such that $V_{2} \sim V_{1}+h H$ as generalized divisor on $S$, where $H$ is the plane section of $S$. This is equivalent to saying there exist surfaces $T_{1}$, of degree $t_{1}$ containing $V_{1}$, and $T_{2}$, of degree $t_{2}$ containing $V_{2}$, such that $t_{2}=t_{1}+h$ and the scheme $W_{1}$ linked to $V_{1}$ by $T_{1} \cap S$ is equal to the scheme $W_{2}$ linked to $V_{2}$ by $T_{2} \cap S$ (see Proposition 4.3 (b) in [21], or [26], pg. 276) 
Thus, we can state the following:

Proposition 6.11. Let $\left[s_{0}\right] \in \mathcal{V}_{\delta}(\mathcal{E}(k))$ with $\mathcal{E}$ a non-splitting, globally generated rank-two vector bundle on $\mathbb{P}^{3}$ and with $k$ and $\delta$ positive integers such that $\delta \leq k+1$. Let $C=V\left(s_{0}\right)$ and $\Sigma=\operatorname{Sing}(C)$. Then, for each integer $h \geq 1$ as in (6.4), each curve $\Gamma_{h}$ as in Theorem 6.3 (i) is obtained from $C$ by an elementary biliaison of height $h$ on the surface $S_{k, h}$

Proof. For simplicity of notation, we denote by $S$ the surface $S_{k, h}$. Denote by $\mathcal{G}$ the cokernel of the map

$$
\tau=\left(s_{0}, s_{h}\right): \mathcal{O}_{\mathbb{P}^{3}} \oplus \mathcal{O}_{\mathbb{P}^{3}}(-h) \rightarrow \mathcal{E}(k),
$$

where $s_{0} \in H^{0}(\mathcal{E}(k))$ and $s_{h} \in H^{0}(\mathcal{E}(k+h))$ are such that $C=V\left(s_{0}\right)$ and $\Gamma_{h}=$ $V\left(s_{h}\right)$. By the diagram

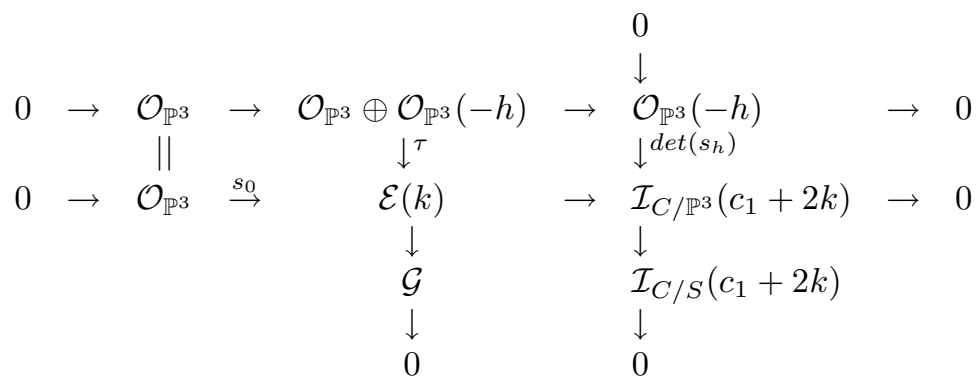

we see that $\mathcal{G} \cong \mathcal{I}_{C / S}\left(c_{1}\right)$. Reversing the roles of $s_{0}$ and $s_{h}$ in diagram (6.12), we similarly find that $\mathcal{G} \cong \mathcal{I}_{\Gamma_{h} / S}\left(c_{1}+h\right)$. Hence, we get $\mathcal{I}_{C / S} \cong \mathcal{I}_{\Gamma_{h} / S}(h)$; since $\mathcal{E}$ is non-splitting, neither $C$ nor $\Gamma_{h}$ can be equivalent to multiples of the hyperplane sections of $S$. Thus, by Definition 6.10 $\Gamma_{h}$ is obtained by an elementary biliason of height $h$ on $S$.

\section{ACKNOWLEDGMENTS}

Part of this paper was prepared during my visit to the Department of Mathematics of the University of Illinois at Chicago (February - May 2001). Therefore, my deepest gratitude goes to L. Ein, not only for the organization of my visit, but mainly for all I have learnt from him, for having suggested that I approach this problem, as well as for the valuable talks we had together. I am greatful to my colleagues (and friends) G. Bini, N. Budur and T. de Fernex, for all that they organized during my visit and with whom I had some informal, but fundamental, talks. My warm thanks go to the Departments of Mathematics of the Universities of Illinois at Chicago, and also to the University of Michigan at Ann Arbor, for making my period of study and research enjoyable, lively and productive. I am indebted to L. Chiantini, C. Ciliberto and the first referee of this paper for their remarks concerning Proposition 6.11. My very special thanks go to GNSAGA-INdAM and to V. Barucci, A. F. Lopez and E. Sernesi for their confidence and their support during my visit to the U.S.A.

\section{REFERENCES}

1. Ballico E., Chiantini L., A look into the Severi varieties of curves in higher codimension. Dedicated to the memory of Fernando Serrano, Collect. Math., 49 (1998), no. 2-3, 191-201. MR 2000h:14025 
2. Barth W., Peters C., Van de Ven A., Compact Complex Surfaces, Springer, Berlin, 1984. MR 86c:32026

3. Beltrametti M., Sommese A.J., Zero cycles and $k$-th order embeddings of smooth projective surfaces (with an appendix by L. Göttsche), in Problems in the theory of surfaces and their classifications (F. Catanese, C. Ciliberto, M. Cornalba, eds.), Sympos. Math., 32, Accademic Press, London, 1991, 33-48. MR 95d:14005

4. Caporaso L., Harris J., Counting plane curves of any genus, Invent. Math., 131 (1998), 345392. MR 99i:14064

5. Chiantini L., On 4-generated bundles and directly linked subcanonical curves, J. Algebra, 99 (1986), 239-262. MR 87g:14027

6. Chiantini L., Ciliberto C., On the Severi varieties of surfaces in $\mathbb{P}^{3}$, J. Algebraic Geom., 8 (1999), 67-83. MR 2000f:14082

7. Chiantini L., Lopez A. F., Focal loci of families and the genus of curves on surfaces, Proc. Amer. Math. Soc., 127 (1999), no.12, 3451-3459. MR 2000e:14057

8. Chiantini L., Madonna C., ACM bundles on a general quintic threefold, preprint math.AG/0110102v1 (2001).

9. Chiantini L., Sernesi E., Nodal curves on surfaces of general type, Math. Ann., 307 (1997), 41-56. MR 98b:14026

10. de Cataldo, M.A.A., Effective nonvanishing, effective global generation, Ann. Inst. Fourier (Grenoble), 48 (1998), no. 5, 1359-1378. MR 99m:14007

11. Demailly J.P., Singular Hermitian metrics on positive line bundles, in Complex Algebraic Varieties, Proc. 1990, Bayreuth, Lectures Notes in Math., 1507, Springer, Berlin, 1992, 87104. MR 93g:32044

12. Ein L., Küchle O. \& Lazarsfeld R., Local positivity of ample line bundles, J. Differential Geom., 42 (1995), no. 2, 193-219. MR 96m:14007

13. Flamini F., Families of nodal curves on projective surfaces, Ph.D thesis, Consortium Universities of Rome "La Sapienza" and "Roma Tre", (2000). Available on http://www.mat.uniroma3.it/dipartimento/esterni/flamini_ricerca.html

14. Flamini F., Moduli of nodal curves on smooth surfaces of general type, J. Algebraic Geom., 11 (2002), no. 4, 725-760.

15. Friedman R., Algebraic surfaces and holomorphic vector bundles, Springer-Verlag, New York, 1998. MR 99c:14056

16. Greco, S., Remarks on the postulation of zero-dimensional subschemes of projective space, Math. Ann., 284 (1989), no.2, 343-351. MR 90f:14002

17. Greuel G.M., Lossen C., Shustin E., Geometry of families of nodal curves on the blown-up projective plane, Trans. Amer. Math. Soc., 350 (1998), no.1, 251-274. MR 98j:14034

18. Harris J., On the Severi problem, Invent. Math., 84 (1986), 445-461. MR 87f:14012

19. Hartshorne R., Ample Subvarieties of Algebraic Varieties, Springer LNM, 156, SpringerVerlag, Berlin, 1970. MR 44:211

20. Hartshorne R., Algebraic Geometry (GTM No. 52), Springer-Verlag, New York - Heidelberg, 1977. MR 57:3116

21. Hartshorne R., Generalized divisors on Gorenstein schemes, K-Theory, 8 (1994), 287-339. MR 95k:14008

22. Horrocks G., Mumford D., A rank-two vector bundle on $\mathbb{P}^{4}$ with 15,000 symmetries, Topology, 12 (1973), 63-81. MR 52:3164

23. Kawachi T., Maşek V., Reider-type theorems on normal surfaces, J. Algebraic Geom., 7 (1998), 239-249. MR 99f:14040

24. Küchle O., Multiple point Seshadri constants and the dimension of adjoint linear series, Ann. Inst. Fourier (Grenoble), 46 (1996), no. 1, 63-71. MR 97d:14010

25. Okonek C., Schneider M., Spindler H., Vector bundles on complex projective spaces, Progress in Mathematics, 3, Boston-Basel-Stuttgart, Birkhäuser, 1980. MR 81b:14001

26. Lazarsfeld R., Rao P., Linkage of general curves of large degree, in Algebraic Geometry - Open Problems (Ravello 1982), Lecture Notes in Math., 997, Springer, Berlin 1983, 267-289. MR 85d:14043

27. Lichtenbaum S. Schlessinger M., The cotangent complex of a morphism, Trans. Amer. Math. Soc., 128 (1967), 41-70. MR 35:237

28. Miyaoka Y., Peternell T., Geometry of higher dimensional algebraic varieties, DMV-Seminar, Bd. 26, Birkhäuser, Basel, 1997. MR 98g:14001 
29. Ran Z., On nodal plane curves, Invent. Math., 86 (1986), 529-534. MR 87j:14039

30. Rao A.P., Liaison among curves in $\mathbb{P}^{3}$, Invent. Math., 50 (1979), 205-217. MR 80e:14023

31. Reider I., Vector bundles of rank 2 and linear systems on algebraic surfaces, Ann. of Math. (2), 127 (1988), 309-316. MR 89e:14038

32. Sernesi E., On the existence of certain families of curves, Invent. Math., 75 (1984), 25-57. MR 85e:14035

33. Severi F., Vorlesungen über algebraische Geometrie, Teubner, Leipzig, 1921. MR 39:6880 (reprint)

34. Shiffman B., Sommese A.J., Vanishing Theorems on Complex Manifolds, Progress in Mathematics, 56, Boston-Basel-Stuttgart, Birkhäuser, 1985. MR 86h:32048

35. Szpiro L., Lectures on equations defining space curves, TATA Institute of fundamental research, Bombay, Springer, 1979. MR 82d:14017

36. Xu G., Subvarieties of general hypersurfaces in projective space, J. Differential Geom., 39 (1994), 139-172. MR 95d:14043

Dipartimento di Matematica, Universitá degli Studi "Roma Tre", Largo San Leonardo Murialdo, 1 - 00146 Roma, Italy

Current address: Dipartimento di Matematica, Universitá degli Studi di L'Aquila, Via VetoioLoc. Coppito, 67010 L'Aquila, Italy

E-mail address: flamini@matrm3.mat.uniroma3.it 